\title{
Distribution and surface enrichment of radionuclides in lead-bismuth eutectic from spallation targets ${ }^{\star}$
}

\author{
Bernadette Hammer-Rotzler ${ }^{1,2}$, Jörg Neuhausen², Viktor Boutellier ${ }^{2}$, Michael Wohlmuther ${ }^{2}$, L. Zanini ${ }^{3}$, J.-C. David ${ }^{4}$, \\ Andreas Türler ${ }^{1,2}$, and Dorothea Schumann²,a \\ 1 University of Bern, 3012 Bern, Switzerland \\ 2 Paul Scherrer Institute, 5232 Villigen PSI, Switzerland \\ 3 European Spallation Source ESS AB, P.O. Box 117, SE-22100 Lund, Sweden \\ ${ }^{4}$ CEA Saclay, Irfu/SPhN, 91191 Gif-sur-Yvette, Cedex, France
}

Received: 21 August 2015 / Revised: 22 April 2016

Published online: 15 July 2016 - (C) Società Italiana di Fisica / Springer-Verlag 2016

\begin{abstract}
With the development of new high-power neutron spallation sources - both for scientific application and as neutron production tool for accelerator-driven systems - the demand for experimentally obtained nuclear data on the residue nuclei production in the target is constantly increasing. In the present work, we examined two lead-bismuth-eutectic targets, irradiated with high-energy protons, concerning their radionuclide content and the spatial distribution of selected isotopes. The first one was the so-called ISOLDE target, being irradiated with 1-1.4 GeV protons at CERN-ISOLDE, the second one was the MEGAPIE target, irradiated at PSI with $590 \mathrm{MeV}$ protons. In particular, we investigated the phenomenon of radionuclide enrichment on free surfaces in both targets. It turned out that considerable accumulation can be found especially in the case of lanthanides. The depletion process is enhanced at increased temperatures. The results are compared with theoretical predictions; some possible consequences of the findings are illustrated.
\end{abstract}

\section{Introduction}

Liquid metals, such as lead-bismuth-eutectic (LBE) or lead, are considered to be used in future fast reactors as spallation target and/or coolant. However, an industrial large-scale application of these materials in such a facility requires careful fundamental and advanced studies concerning their performance under operational as well as accidental conditions, completed by an appropriate waste management.

One of the pioneering experiments for testing liquid metals as spallation target material under high-power conditions was MEGAPIE (MEGAwatt PIlot Experiment) — a joint initiative by six European research institutions, the USA, Korea and Japan to design, build, operate and explore a liquid-metal lead-bismuth-eutectic (LBE) spallation target of $1 \mathrm{MW}$ beam power [1]. After its successful operation in 2006 in the Spallation Neutron Source facility SINQ at PSI, using the proton accelerator HIPA (590 MeV, continuous flux) as a driver, the target was cut into pieces and sampled for the Post-Irradiation Examination (PIE) [2]. Besides around 800 samples, planned for material research investigations at the partner institutes, also 76 samples were taken from the LBE bulk, the steel-LBE interface and the free LBE surface, i.e. the interface with the cover gas. Additionally, several samples from an absorber, installed into the expansion tank for removing volatiles, were taken. With this stock of sample material, representing a unique archive of information on the production as well as the chemical and physico-chemical behavior of radionuclides in a high-power spallation target, a series of extended radioanalytical examinations was performed, aimed at gaining knowledge about production rates, accumulation and depletion processes as well as benchmarking nuclear codes and models in order to improve the quality of predictions. Special attention was paid to radionuclides with a high radiological risk due to their nuclear and/or chemical properties like high solubility or volatility, making them hazardous to the environment. In total, 20 radionuclides were determined and quantified. It turned out that bulk LBE contains mainly noble metals that have a significant solubility in LBE (gold, mercury, silver, rhodium), while radionuclides of elements that are sensitive

\footnotetext{
* Contribution to the Focus Point on "Nuclear data for energy" edited by S. Leray.

a e-mail: dorothea.schumann@psi.ch
} 
to oxidation/reduction are accumulated in samples taken at the interfaces. For most of the determined radionuclides, a good or fair agreement of the total content with theoretical predictions was found. The outcome of these analyses was already published previously in several papers [3-6] and compiled in the PhD thesis of Hammer-Rotzler [7].

During the preparation of MEGAPIE, several fundamental studies concerning the release behavior of hazardous radionuclides [8-15] were carried out to ensure the safe operation of the experiment. One of them was the so-called ISOLDE experiment, performed at CERN-ISOLDE. Solid LBE, filled into the standard ISOLDE container, was molten and then irradiated by $1-1.4 \mathrm{GeV}$ protons to study the production and volatilization of radionuclides online [12-15]. After irradiation, the target was shipped to PSI and radiochemical examinations were performed to determine the radionuclide inventory and to study the physico-chemical behavior of the selected radionuclides.

In the present work, we report on investigations on these ISOLDE samples as well as on selected samples from the LBE/cover gas-interface of the MEGAPIE target, performed using $\gamma$ - and $\alpha$-spectrometry. For the determination of radionuclides which cannot be measured in the initial sample by $\gamma$-spectrometry, radiochemical separation procedures were developed.

\section{Experimental part}

\subsection{ISOLDE target}

\subsubsection{Irradiation}

The target was irradiated at the ISOLDE facility (Isotope Separator On-Line Detector) at CERN in support of the MEGAPIE Project to study the production and volatilization of radionuclides in liquid LBE targets. The irradiation was performed in two runs in 2004 and 2005, lasting about 10 days each [13]. Between 28th April and 5th May 2004 the target received $1.3 \times 10^{17}$ protons of a pulsed beam of $1.4 \mathrm{GeV}$ and the production and release of volatiles was studied at 400 and $600^{\circ} \mathrm{C}$ (melting point of LBE: $125^{\circ} \mathrm{C}$ ). Between 15th and 23rd August 2005 the target took another $1 \times 10^{18}$ protons of $1 \mathrm{GeV}$ and $1.4 \mathrm{GeV}$. In this second run, the operating temperature was varied between 250 and $600{ }^{\circ} \mathrm{C}$, with most of the run performed at the highest temperatures. Afterwards the target was stored at CERN until it was transported to PSI in 2008 for radiochemical studies. Large quantities of gaseous and highly volatile elements, such as noble gases or mercury, were not expected to be present in the target material because it was heated under vacuum when irradiated, leading to fast evaporation $[12,13,15]$. Because of that, only the quantitative determination of less volatile radionuclides was possible. The ISOLDE LBE target lends itself to distribution studies of radioactive elements and is an excellent model for the development of the separation system for analysis of samples from MEGAPIE.

\subsubsection{Sample taking}

The ISOLDE target was transported from CERN to the PSI Hotlab where it was cut and samples were prepared for further analysis. The spallation target consisted of a cylindrical tantalum container of $20 \mathrm{~cm}$ length and $1 \mathrm{~cm}$ radius, which was filled to about $75 \%$ vol. with liquid LBE [13]. The mass of the LBE was 547 grams (fig. 1). Using a saw, the target containing the now solidified LBE was cut into five pieces, namely target bottom, T1, T3, T2 and target head. The holder at the target head and at the target bottom were cut and removed. For radiochemical separation and analyses, the samples T1-C-bulk-disc, T1-1C-bulk, T2-1H-bulk, T2-1C-surface and T2-1C-bulk (C = Chimney side; $\mathrm{H}=$ Head side) were used (see table 1). Their locations in the target are illustrated in fig. 1.

\subsection{MEGAPIE target}

\subsubsection{Target assembly}

Figure 2 shows a cutaway and schematic view of the MEGAPIE target. The dimensions of the target are $5.35 \mathrm{~m}$ in length, with a lower diameter of $200 \mathrm{~mm}$ and an upper diameter of $400 \mathrm{~mm}$. The target is composed of the upper target enclosure (10), lower target enclosure (7), upper liquid metal container (B) and lower liquid metal container (8, A). The major components are the target heat exchanger $(5, \mathrm{c})$, the electromagnetic pumps $(4, \mathrm{~d}$, e), the target head (a), the target top shielding (b), the central rod (f) and the main and the bypass flow guide tube (g).

The lower liquid metal container is one of the most critical structural parts of the MEGAPIE target, because the beam window, a steel hemi-sphere cap (2), is exposed to intense proton and neutron irradiation, thermal and mechanical load and the presence of the flowing LBE. For the structural material of the lower liquid metal container T91 steel was used because of its resistance to radiation damage, whereas for the upper part 316L steel was chosen because of its corrosion resistance. The highest temperature gradient in the system occurs along the heat exchanger. 


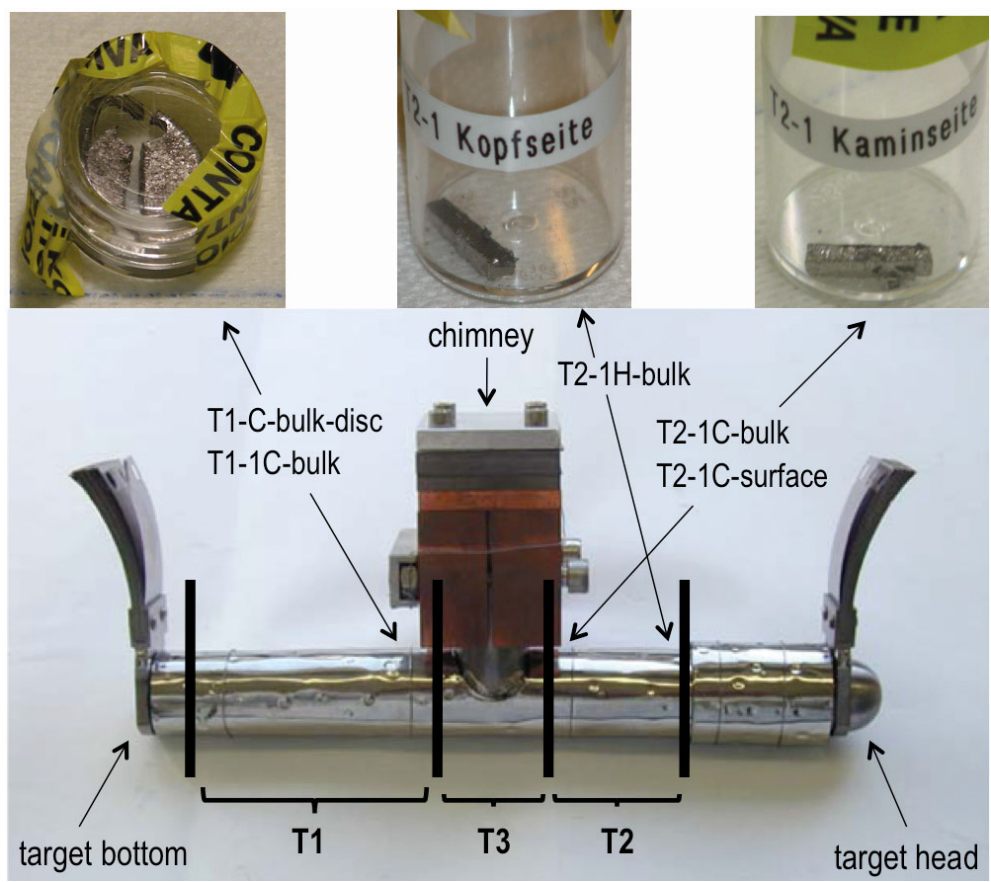

Fig. 1. ISOLDE target; proton beam enters from the target head.

Table 1. Summary of analyzed sample types from ISOLDE target and their characteristics.

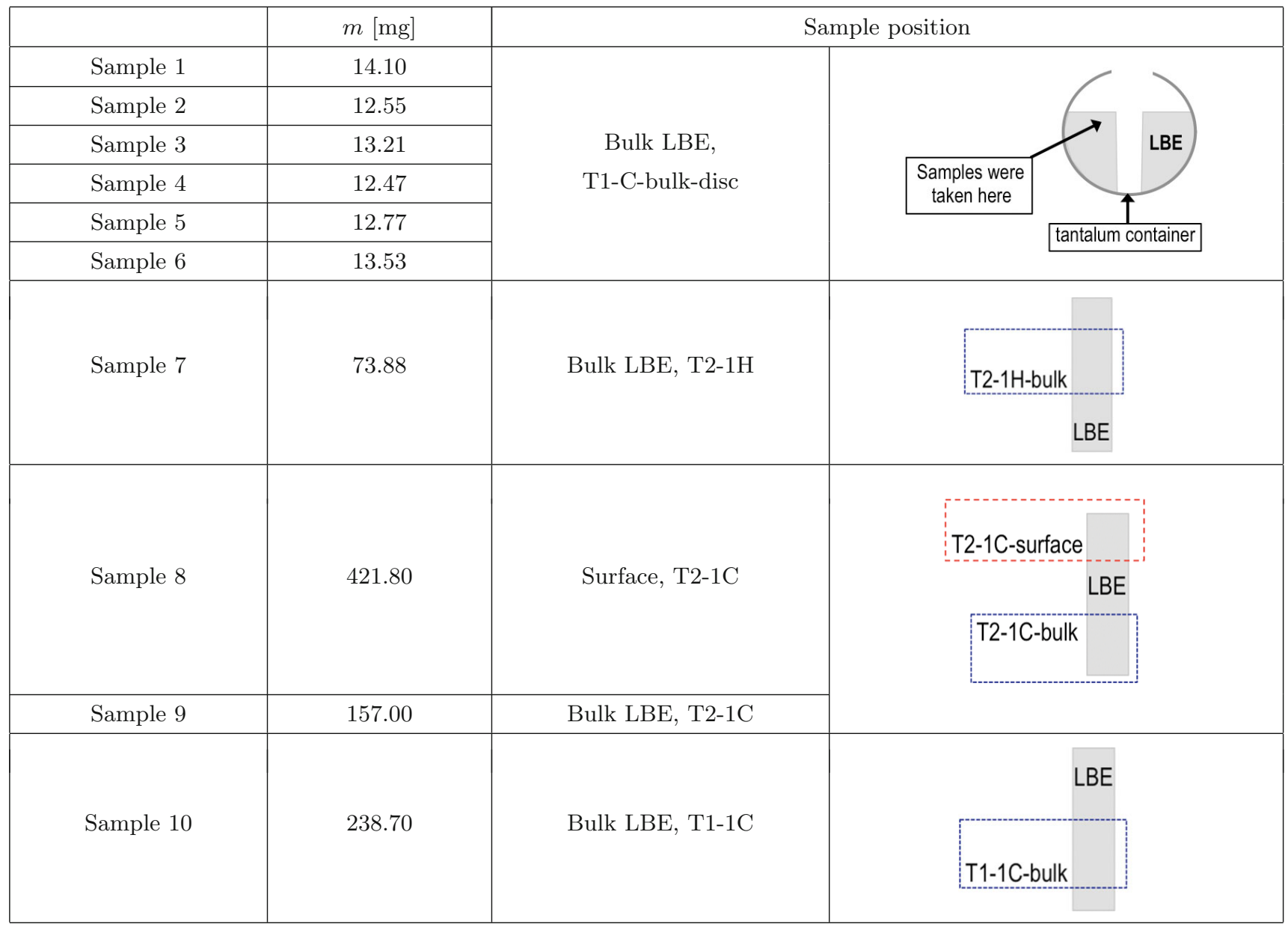




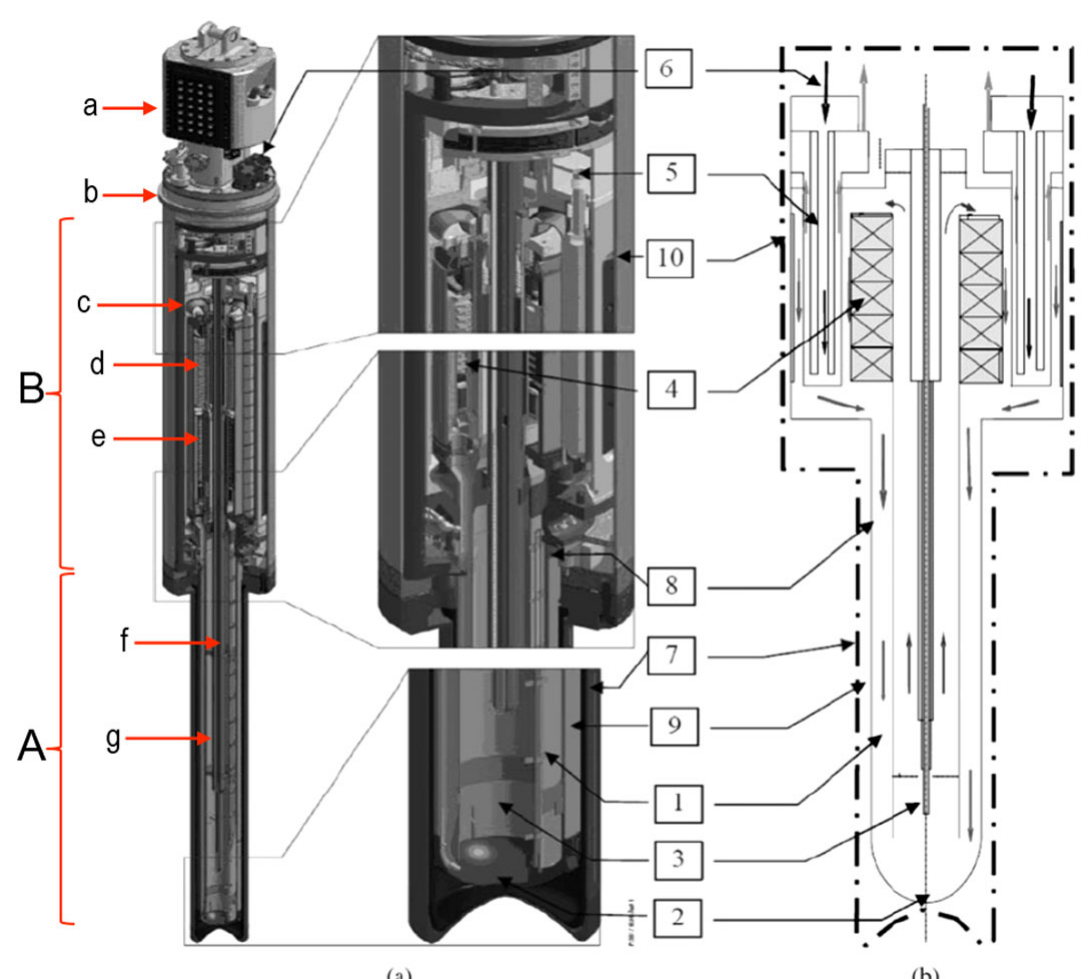

(a)

(b)

Fig. 2. MEGAPIE target in (a) cutaway and (b) schematic representation: (1) lower liquid metal container outer annulus; (2) lower liquid metal container window; (3) lower liquid metal container rise channel; (4) electromagnetic pumps; (5, c) Diphyl oil heat exchanger; (6) Diphyl oil in/out; (7) lower target enclosure; (8, A) lower liquid metal container; (9) helium-filled isolation gap; (10) upper target enclosure; (B) upper liquid metal container; (a) target head; (b) shielding; (d) main electromagnetic pump; (e) bypass electromagnetic pump; (f) central rod; (g) main and bypass flow guide tube. Reproduced from [16]. Copyright 2008 by the American Nuclear Society, La Grange Park, Illinois.

The lower and upper parts of the target are connected with a flange system. The wall thickness of the lower part and the upper part is $2 \mathrm{~mm}$ and $4 \mathrm{~mm}$, respectively.

The coolant is pumped through the target (max. LBE flow velocity $1 \mathrm{~m} / \mathrm{s}$ ) by the main electromagnetic pump (d), located between the annular heat exchanger and an internal cylindrical guide tube. Thus, the pump separates the hot and cold LBE streams $\left(230-350^{\circ} \mathrm{C}\right)$. LBE is discharged from the heat exchanger to the annular space between the guide tube and the target hull with a temperature of $230^{\circ} \mathrm{C}$, where it descends to the window and then rises in the space inside the guide tube towards the main electromagnetic pump entrance. Here, the hot LBE, heated by the proton beam to temperatures around $380^{\circ} \mathrm{C}$, is pumped through the inlet manifold of the heat exchanger and down over 12 cooling pins to complete the LBE circuit. A bypass pump (e) is situated beneath the main pump, which is used to provide a secondary coolant flow to the window. The combination of main flow and bypass flow ensures the proper cooling of the beam window. In the center of the target there is a central rod, which also contains an electrical heater for maintaining the LBE temperature during start-up and shut-down periods. The LBE target is surrounded by a $\mathrm{D}_{2} \mathrm{O}$-cooled safety vessel, which is intended to protect the proton beam tube from possible LBE leaks. The target assembly is mounted vertically, and the proton beam penetrates the target from the bottom through the safety hull and the target window up to a depth of $30 \mathrm{~cm}$ of the target [16], generating $10^{17}$ high-energy spallation neutrons per second [17]. The MEGAPIE cooling system is of a triple-loop type, employing three working fluids: LBE (primary coolant), Diphyl oil (intermediate cooling loop) and heavy water (secondary cooling system). The oil loop is advantageous to avoid any possible LBE/water contact and the loops are thermally connected by heat exchangers. In the topmost part of the target, the LBE loop was connected to an expansion volume, where the liquid metal was in contact with a cover gas (Ar). This interface of the LBE with the cover gas is also referred to as "free surface" of the LBE.

\subsubsection{Irradiation}

The MEGAPIE target was irradiated with an average current of $0.947 \mathrm{~mA}$ of $575 \mathrm{MeV}$ protons for 123 days. Before operation, the molten LBE filled into the target system was kept in the liquid state using heaters. The temperature 


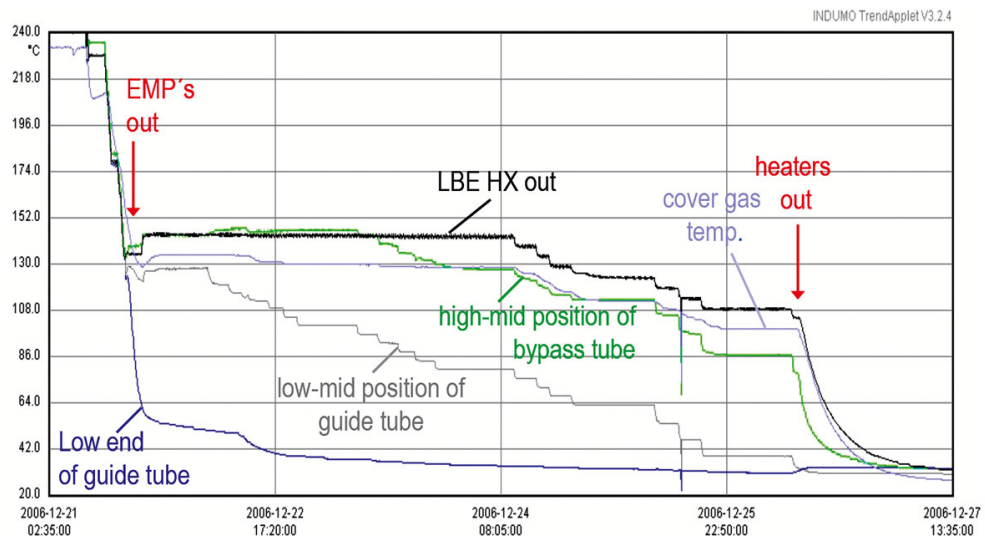

Fig. 3. History of temperatures in MEGAPIE target during cool down and freezing of the LBE after irradiation (EMPs: Electromagnetic Pumps; HX: Heat exchanger) [20].

was regulated with thermocouples, distributed within the target. In the reaction zone in the lower part of the target, the LBE was heated by the proton beam to temperatures higher than $340{ }^{\circ} \mathrm{C}$ to $350{ }^{\circ} \mathrm{C}[18,19]$. From this zone, the liquid metal moved upwards through the electromagnetic pump until it was diverted close to the top of the target to flow down again through the heat exchanger, being cooled down to $230^{\circ} \mathrm{C}$ [18] and finally returning to the reaction zone. In the topmost part of the target, the LBE loop was connected to an expansion volume, where the liquid metal was in contact with argon as cover gas. The mass transfer between the loop and this expansion volume was enhanced by level changes of the liquid metal caused by thermal expansion and contraction of the LBE with changing operating conditions (temperature changes induced by beam trips and switching between standby and full power operation modes). The major part of the proton-induced nuclear reactions took place in the irradiation zone (up to $30 \mathrm{~cm}$ above the window). The nuclear reaction products formed in this zone were then circulated through the target system by the liquid metal flow, being exposed to thermal gradients and coming in contact with interfaces of the structural materials and the cover gas. On December 21st in 2006 the proton beam was stopped and the target was allowed to cool down. For this purpose the heat removal system was regulated in such a way that the temperature of the LBE dropped from $230^{\circ} \mathrm{C}$ to $140^{\circ} \mathrm{C}$. After reaching a homogeneous temperature of $140^{\circ} \mathrm{C}$ the electromagnetic pumps (EMPs) were switched off and the LBE was no longer in circulation (fig. 3). Afterwards the LBE temperature was reduced in the lower target position by gradually lowering the temperature of the central rod heaters, while keeping the upper LBE temperature above the melting point by circulating the hot oil through the target heat exchanger (HX) pins using the heat removal system. In this way, the LBE was frozen from the bottom-up, avoiding adverse effects caused by volume expansion of the solidifying LBE. Around New Year 2007 the LBE was solid [20].

\subsubsection{MEGAPIE sampling}

After a cooling time of 4 years the target was cut into segments as shown in fig. 4. Samples for radiochemical analyses were taken from the segments H02 to H09. In total, we retrieved 76 samples to facilitate conclusions about the distribution of radionuclides in the target. 32 of these samples were taken from the LBE/steel and LBE/cover gasinterfaces to study deposition or enrichment effects of these interfaces. The remaining 43 samples represent the bulk LBE. In this article, we will address only the samples from the LBE-cover gas-interface. This part of the sampling will be explained in the following section. A detailed explanation of the entire sample taking as well as results of radiochemical analysis of samples from the other parts of the MEGAPIE target can be found in [3-7].

\subsubsection{Sampling the LBE/cover gas-interface of MEGAPIE}

During operation, the free surface of the LBE was located in the lower part of the expansion volume, between the sections H08 and H09 (see fig. 4). After the end of irradiation, the level of this free surface declined, leaving the segments $\mathrm{H} 08$ and H09 virtually free of LBE, apart from some material that was sticking on the wall. Visual inspection showed that two different kinds of material are located in this region of the target, one consisting of solidified LBE that is contaminated with a black solid (fig. 5), whereas the other consists of a grey and yellow colored solid material sticking mainly to the central rod (fig. 6). One sample from each type of material was analyzed (H08-U2 and H08-U1b). Furthermore, two samples from segment H07 were analyzed, H07-U2 and H07-S (figs. 7 and 8). Sample H07-U2 represents material that is similar in nature to sample H08-U2, i.e. it consists of LBE contaminated with a dark solid. 


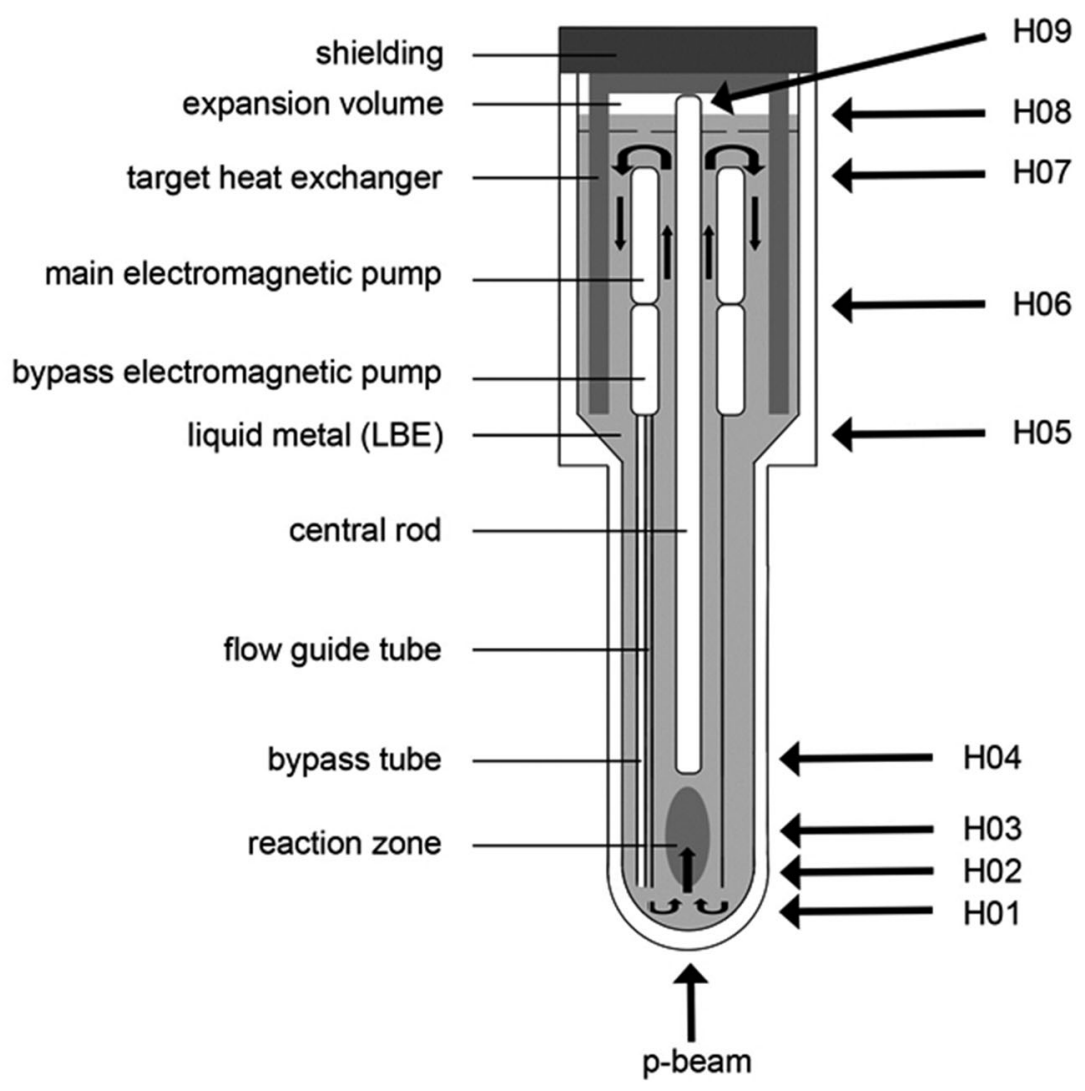

Fig. 4. Schematic view of the MEGAPIE target assembly and its main components. The location of the segments Hxx used for the post-irradiation examination are indicated on the right. Figure reproduced from [3].

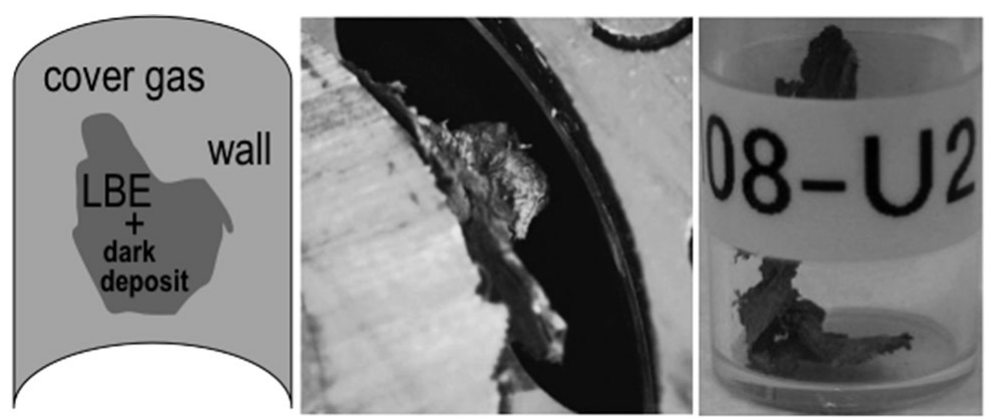

Fig. 5. Sampling of an LBE/cover gas-interface sample, H08-U2 that was sticking to the wall. Left: schematic view; middle: sample, H08-U2, sticking to the wall; right: the final sample. Figure reproduced from [6].

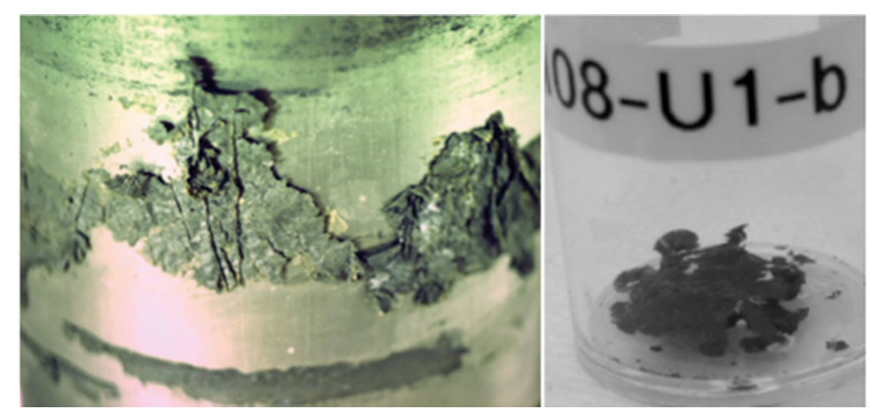

Fig. 6. Sampling of LBE/cover gas-interface sample H08-U1-b. This sample consists of grey and yellow coloured solid material that had the appearance of a powder that is sintered to form a crust sticking to the steel wall. Figure reproduced from [6]. 


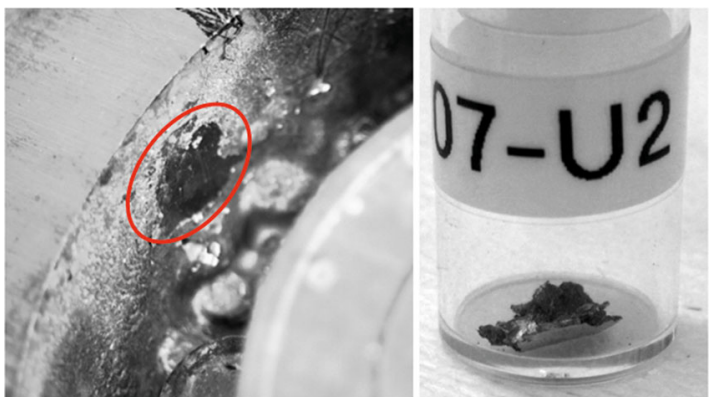

Fig. 7. Sampling of H07-U2. The sample consists of LBE that is contaminated with a dark solid material on its surface and was found sticking to the wall of a ring-shaped structure within segment H07. Figure reproduced from [6].

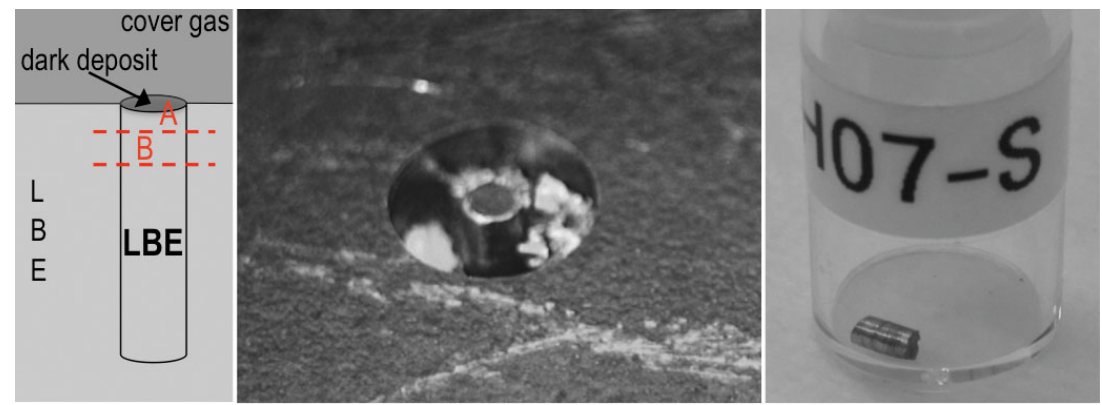

Fig. 8. Sampling of an LBE/cover gas-interface sample, H07-S. Note the black layer visible on the free LBE surface (middle) at the cover gas-interface, indicating the formation of an oxide layer. Left: schematic view showing LBE and the thin black surface layer. A indicates the position of the used sample; middle: drilled sample before breaking off; right: the final sample. Figure reproduced from [6].

Sample H07-S was taken by core drilling and represents the solidified free surface of LBE (fig. 8). The complete surface of the LBE was found to be covered with a thin layer of a black or dark brown solid material. For the analysis, the top part of the cylinder containing the dark deposit was cut using a scalpel. In table 2, the properties of the four analyzed samples from the free surface region are summarized.

\subsection{Chemical separation}

For the separation procedure samples of about $12 \mathrm{mg}$ of material were dissolved in $\mathrm{HNO}_{3}$. The solution was evaporated to dryness and the residue was dissolved in $\mathrm{HCl}(35 \%-37 \%)$ and again evaporated to dryness. This procedure was repeated at least twice, in order to remove remaining nitrate. Then, the residue was dissolved in $10 \mathrm{ml}$ of $0.8 \mathrm{M} \mathrm{HCl}$ and used as stock solution. An aliquot of the stock solution was loaded onto a Dowex 50WX8 column. The matrix was eluted with $15 \mathrm{ml} 0.9 \mathrm{M} \mathrm{HCl}$. The lanthanides were eluted with $15 \mathrm{ml} 8 \mathrm{M} \mathrm{HCl}$, and the solution was evaporated to dryness. After re-dissolving the residue in $2 \mathrm{ml} 0.1 \mathrm{M} \mathrm{HNO}_{3}$ and loading it onto a specific LN-resin column, the lanthanide fractions can be separated with $\mathrm{HNO}_{3}$ solutions between $0.4 \mathrm{M}$ and $0.6 \mathrm{M}$. For additional details see [5]. A following electrochemical deposition of ${ }^{148} \mathrm{Gd}$ was performed in i-PrOH $(30 \mathrm{~min}, 500 \mathrm{~V}, \sim 7 \mathrm{~mA})$ with a $\mathrm{Pt}$ electrode onto steel after evaporating the corresponding fraction to dryness and dissolving the residue in $100 \mu \mathrm{l} 0.1 \mathrm{M} \mathrm{HNO}_{3}$. This method provides samples with a thin layer of activity on the surface guaranteeing a sufficient resolution of the recorded $\alpha$-spectra.

For monitoring the chemical yields of the $\alpha$-emitting ${ }^{148} \mathrm{Gd}$, the samples were spiked with $\gamma$-ray-emitting ${ }^{153} \mathrm{Gd}$. For details see [5].

\subsection{Dedicated distribution and surface enrichment studies with ISOLDE samples}

To obtain more information on the radionuclide distribution in the bulk material from the ISOLDE target, a cuboid of bulk LBE from section T2-1H $(m=73.88 \mathrm{mg})$ was cut into 47 sections of $40 \mu \mathrm{m}$ thickness using a microtome (Leica, RM2235). Before cutting, the cuboid was cleaned by repeated cutting of the surfaces of the six sides. For cutting, a standard steel knife was used. After the cutting, $\gamma$-spectra were recorded for each of the 47 specimens.

Additionally, the fact that the range of $\alpha$-particles emitted by the radionuclides present in the LBE is limited (e.g., for the $3.2 \mathrm{MeV} \alpha$-particles emitted by ${ }^{148} \mathrm{Gd}$ the range is approximately $10 \mu \mathrm{m}$ ) can be used to qualitatively study 
Table 2. Summary of analyzed sample types from the LBE/cover gas-interface of MEGAPIE and their characteristics.

\begin{tabular}{|c|c|c|}
\hline & $m[\mathrm{mg}]$ & Material \\
\hline H08-U2 & 14.2 & LBE sticking to the wall, contaminated with black solid on surface \\
\hline H08-U1-b & 12.0 & Grey and yellow solid, sticking on the wall of central rod, contaminated with LBE \\
\hline H07-U2 & 14.7 & LBE sticking to the wall, contaminated with black solid on surface \\
\hline H07-S & 10.3 & $\begin{array}{c}\text { Core drill in free surface, } \varnothing=2 \mathrm{~mm}, l=5 \mathrm{~mm}, \\
\text { LBE cylinder with black solid on top surface. Contaminated LBE }\end{array}$ \\
\hline
\end{tabular}

the surface enrichment of such nuclides. For this purpose, a sample $(m=421.8 \mathrm{mg})$ was cut from segment T2-1C in such a way that one side of the specimen represented the free surface of the LBE, i.e. the surface that formed the interface of LBE with the vacuum during irradiation, while the opposite side of the specimen represented the bulk LBE. The $\alpha$-particles emitted by the different sides of the specimen were then counted in separate measurements in an $\alpha$-chamber to assess surface enrichment of the $\alpha$-emitting gadolinium isotope ${ }^{148} \mathrm{Gd}$.

Furthermore, we studied also qualitatively the tendency of radionuclides to separate from molten LBE, using samples from the ISOLDE target. For this purpose, one LBE sample (T1-1C-bulk, $238.7 \mathrm{mg}$ ), was cut from the bulk LBE, a $\gamma$-spectrum was recorded and also the $\alpha$-particles emitted from the freshly cut surface of LBE were measured for 2 hours. Afterwards, the sample was put in a quartz boat lined with a quartz fleece and was placed inside a fused silica reaction tube in an $\mathrm{H}_{2}$ stream $(50 \mathrm{ml} / \mathrm{min})$. The reducing atmosphere was chosen to avoid the formation of oxides that could significantly influence the behavior of the radionuclides. The sample was then molten for 60 min at temperatures between $500-600^{\circ} \mathrm{C}$ forming a silvery spherical drop. After cooling to room temperature, the spherical drop was measured by $\gamma$ - and $\alpha$-spectrometry again. Additionally, the surface layer accumulated on the LBE specimen during the melting was mechanically separated from the sample, and both the removed surface layer and the remaining LBE were separately measured by $\gamma$-spectrometry.

\subsection{Measurement technique}

\subsection{1 $\gamma$-measurement}

The samples were measured on an HPGe-detector equipped with standard measuring electronics. The measuring time was chosen in such a way that a sufficient counting statistics was achieved. Energy and efficiency calibrations were performed using ${ }^{60} \mathrm{Co},{ }^{152} \mathrm{Eu}$ and ${ }^{137} \mathrm{Cs}$ point sources. For the data processing, the program GENIE2000 (Canberra) was used. For nuclide identification, a special library was created, containing 34 long-lived nuclides (half-lives of 73.8 days to 440 years) and their radioactive daughters that can be formed by nuclear reactions induced by high-energy protons in lead and bismuth. In special cases, manual data processing was carried out as well. For ${ }^{207} \mathrm{Bi}$ and ${ }^{173} \mathrm{Lu}$, the results produced by the automatic evaluation routine of GENIE2000 were compared with the results of manual calculations for a considerable number of samples. The results obtained with the two methods were always in good agreement.

\section{$2.5 .2 \alpha$-measurement}

The $\alpha$-spectroscopy system consisted of an $\alpha$-spectrometer (vacuum chamber and amplifier electronics), $\alpha$-detector (Passivated Implanted Planar Silicon detector (PIPs), Canberra), analog to digital converter (ADC), multi-channel analyzer (MCA) and computer software (Canberra Alpha software). The resolution of the used $\alpha$-detector was specified as $19 \mathrm{keV}$ by the manufacturer. For the MEGAPIE samples used for quantitative analysis we obtained a resolution of $36 \mathrm{keV}$ (averaged value). The efficiency for the quantitative ${ }^{148} \mathrm{Gd}$ measurements in the MEGAPIE samples was $33.9 \%$.

\subsection{Theoretical predictions}

In the following sections, the experimental data obtained by radioanalytical methods for the activities of radionuclides detected in the ISOLDE target material after irradiation will be compared to predicted values obtained from nuclear physics calculations. For this purpose, the total activities of the studied radionuclides predicted to be produced by end of beam obtained from the nuclear physics codes were divided by the mass of the LBE contained in the target. In this way, predicted activity concentrations in the LBE are obtained that correspond to a homogeneous distribution of the produced radionuclides within the LBE.

Activities of nuclei after irradiation of the ISOLDE LBE target and cooling were calculated by means of the INCL4.6-Abla07 spallation model combination and of the CINDER'90 activation code [21]. The spallation model 


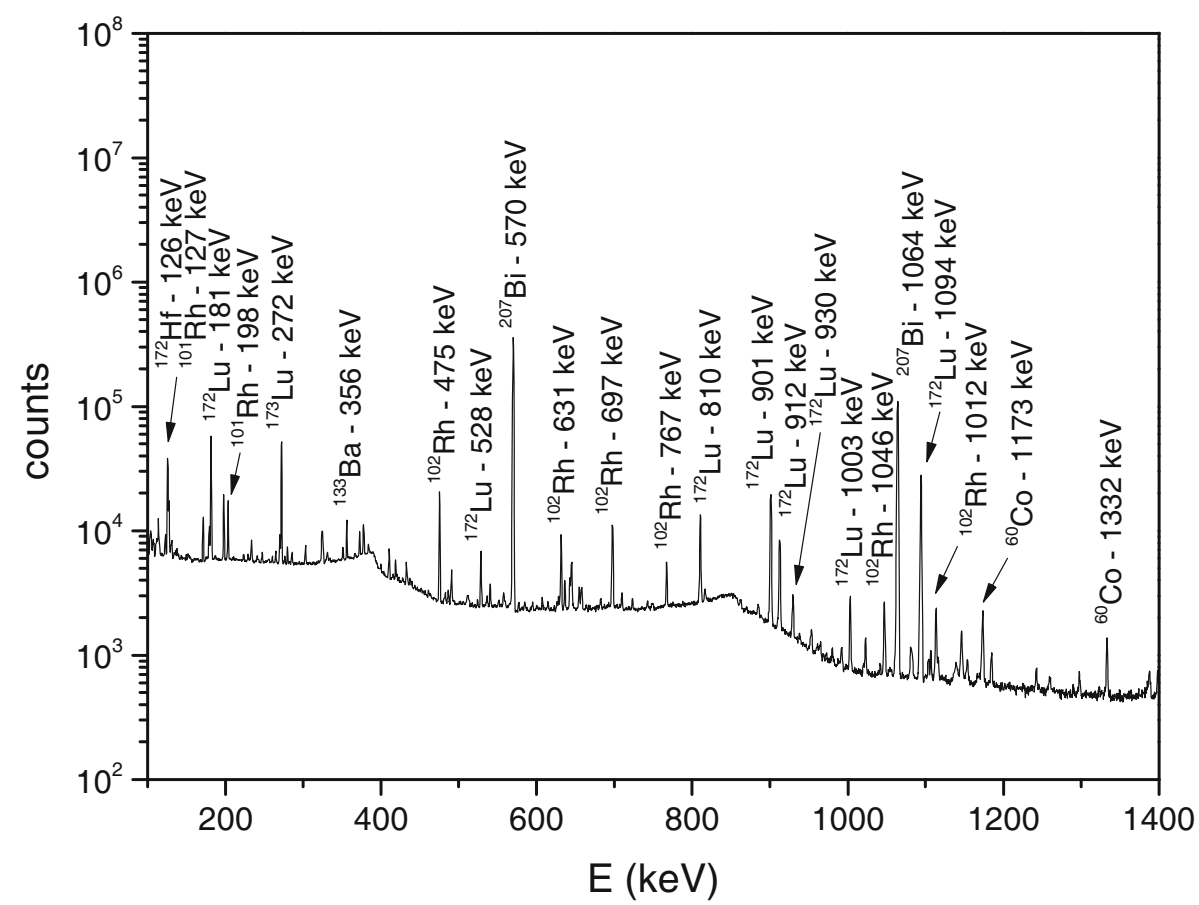

Fig. 9. Typical $\gamma$-spectrum from bulk LBE obtained from the ISOLDE target.

provides nucleus production rates and the activation code their modifications with time (during irradiation and cooling) as well as their activities. The computer code INCL4.6 [22] deals with the first part of the spallation reaction, i.e. the intranuclear cascade, and the Abla07 code [23] describes the de-excitation of the excited remnant nucleus after the end of the cascade. Both were used within the particle transport code MCNPX2.7 (private implementation) in order to take into account the secondary reactions in the target. Nucleons, pions and light nuclei up to alpha were considered as potential projectiles inducing reactions. For comparison, also predicted values from an older calculation reported already elsewhere [24] performed using the Monte Carlo transport code FLUKA [25,26] combined with the evolution code ORIHET3 [27] are given.

\section{Results}

\subsection{ISOLDE target - analysis of $\gamma$-emitters in bulk LBE}

A typical $\gamma$-spectrum of an ISOLDE sample is shown in fig. 9. The results obtained from 6 different bulk LBE samples are compared to the results of theoretical calculations in table 3. Predicted activity concentrations were calculated by relating the total activities predicted to be produced to the mass of LBE present in the target. The values obtained in this way are generally in fair agreement with the experimental results. Only for the Rh isotopes rather strong discrepancies are observed, the INCL/Abla predictions being significantly higher than the analytical results of all samples for ${ }^{101} \mathrm{Rh}$, while the FLUKA predictions are exceptionally high for ${ }^{102} \mathrm{Rh}$. It is also noteworthy that in sample 1 for most of the radionuclides, except ${ }^{207} \mathrm{Bi}$, a significantly larger amount was found experimentally compared to all the other samples. This indicates that in sample 1 an enrichment of radionuclides of elements different from the matrix has occurred.

More information on the distribution of the studied radionuclides in the bulk LBE of the ISOLDE target can be extracted from the results of the microtome cutting study. In figs. 10 and 11, the individual values for the activity concentrations obtained from the single sections are plotted for the studied radionuclides. Table 4 compiles the averages of the activity concentrations over the complete set of 47 sections. Similar to the results obtained for the six individual samples discussed above, the mean values for the measured activity concentrations are in fair agreement with predicted values obtained from nuclear codes assuming homogeneous distribution, except for the two rhodium isotopes, where for ${ }^{101} \mathrm{Rh}$ the INCL/Abla predictions are significantly higher than the analytical data, while for ${ }^{102} \mathrm{Rh}$ FLUKA seems to overestimate its production. From the variation of the activity concentrations throughout the set of 47 individual sections it is obvious that the only nuclide representing the target matrix, ${ }^{207} \mathrm{Bi}$, is practically homogeneously distributed within the LBE, as expected. A similarly homogeneous distribution is also found for $110 m$ Ag. 


\begin{tabular}{|c|c|c|c|c|c|c|c|c|c|}
\hline $\begin{array}{l}2 \\
0 \\
0 \\
0 \\
0 \\
0 \\
0 \\
0\end{array}$ & 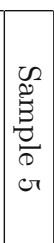 & 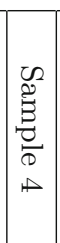 & 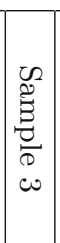 & 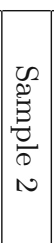 & 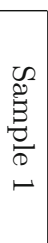 & 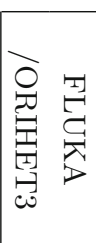 & 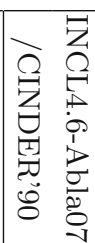 & & \\
\hline \begin{tabular}{|l|}
$\vec{c}$ \\
$\dot{c}$ \\
$\dot{c}$
\end{tabular} & $\begin{array}{l}\vec{v} \\
\stackrel{\vec{v}}{\mathrm{~V}}\end{array}$ & $\mid \begin{array}{l}\vec{N} \\
\vec{N} \\
\vec{v}\end{array}$ & \begin{tabular}{|l|}
$\vec{\omega}$ \\
$\dot{\omega}$ \\
$ن$
\end{tabular} & \begin{tabular}{|l|}
$\vec{N}$ \\
$\dot{d}$ \\
$\mathrm{c}$
\end{tabular} & $\begin{array}{l}\overrightarrow{+} \\
\dot{\overrightarrow{0}}\end{array}$ & & & $\begin{array}{c}3 \\
3 \\
00\end{array}$ & \\
\hline 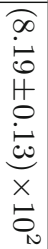 & 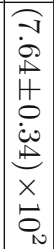 & 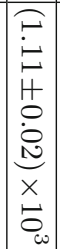 & 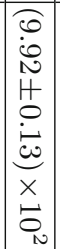 & 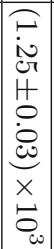 & 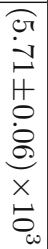 & $\begin{array}{l}\stackrel{N}{*} \\
\dot{\sigma} \\
\times \\
\hat{\sigma}_{\omega}\end{array}$ & $\begin{array}{l}\vec{\sim} \\
\underset{j}{J} \\
\times \\
\underset{\omega}{0}\end{array}$ & $\stackrel{8}{8}$ & \\
\hline 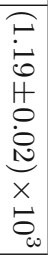 & 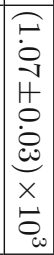 & 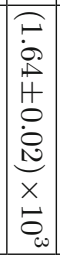 & 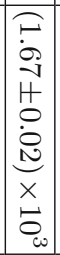 & 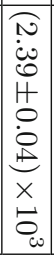 & 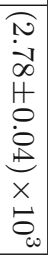 & $\begin{array}{l}c r \\
\dot{\infty} \\
\dot{x} \\
\stackrel{\omega}{\sigma}\end{array}$ & $\begin{array}{l}0 \\
\dot{1} \\
\infty \\
\times \\
\sigma_{\omega}\end{array}$ & $\stackrel{\overrightarrow{0}}{\stackrel{b}{\sigma}}$ & \\
\hline 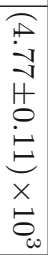 & 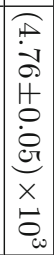 & 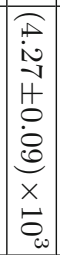 & 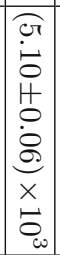 & 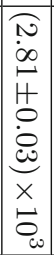 & 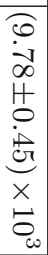 & $\begin{array}{l}\stackrel{0}{0} \\
\stackrel{N}{N} \\
\times \\
ث \\
0\end{array}$ & $\begin{array}{l}\dot{v} \\
\dot{\omega} \\
\dot{\omega} \\
\times \\
\dot{\omega}_{\omega}\end{array}$ & 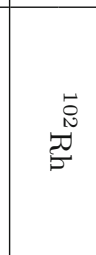 & $>$ \\
\hline 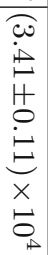 & 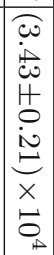 & 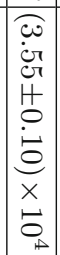 & 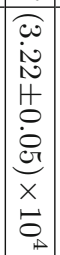 & 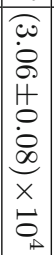 & 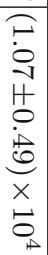 & & & $\underset{\infty}{\stackrel{\overrightarrow{3}}{3}}$ & 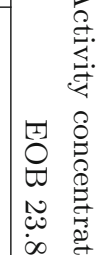 \\
\hline 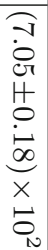 & 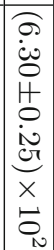 & 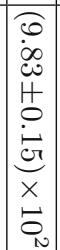 & 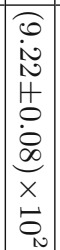 & 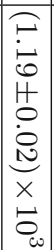 & 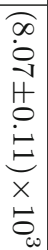 & 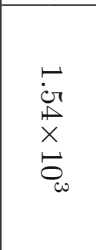 & 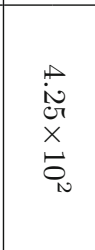 & $\underset{\stackrel{\omega}{\omega}}{\stackrel{\vec{\omega}}{\uplus}}$ & 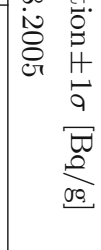 \\
\hline 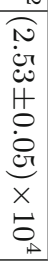 & 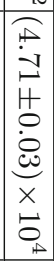 & 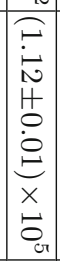 & 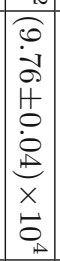 & 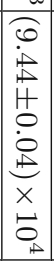 & 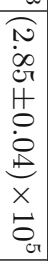 & $\begin{array}{l}\vec{\omega}_{\vec{\omega}} \\
\times \\
\vec{\sigma}_{0}\end{array}$ & 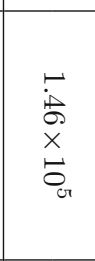 & 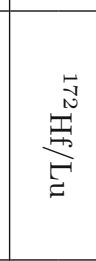 & \\
\hline 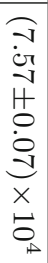 & 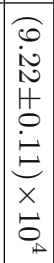 & 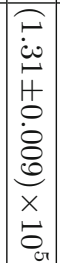 & 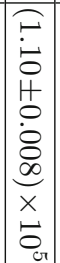 & 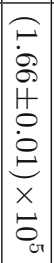 & 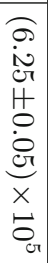 & 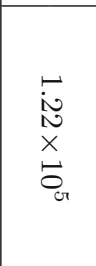 & 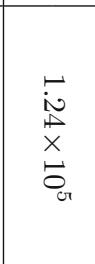 & $\stackrel{\vec{\omega}}{\stackrel{\vec{\omega}}{\leftrightarrows}}$ & \\
\hline 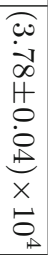 & \begin{tabular}{|c|c|}
0 \\
0 \\
0 \\
1 \\
$1+$ \\
0 \\
0 \\
0 \\
$\times$ \\
$\times$ \\
0 \\
0 \\
+
\end{tabular} & 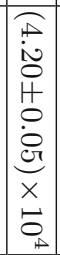 & 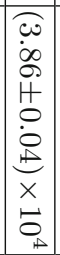 & 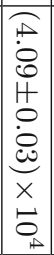 & 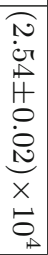 & $\begin{array}{l}0 \\
\dot{0} \\
+ \\
\times \\
\vdots \\
0\end{array}$ & $\begin{array}{l}0 \\
0 \\
0 \\
\times \\
\times \\
0 \\
0\end{array}$ & 苂 & \\
\hline
\end{tabular}

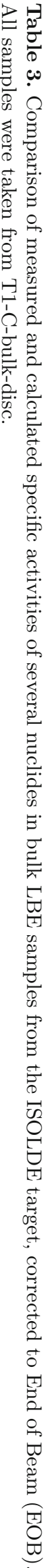




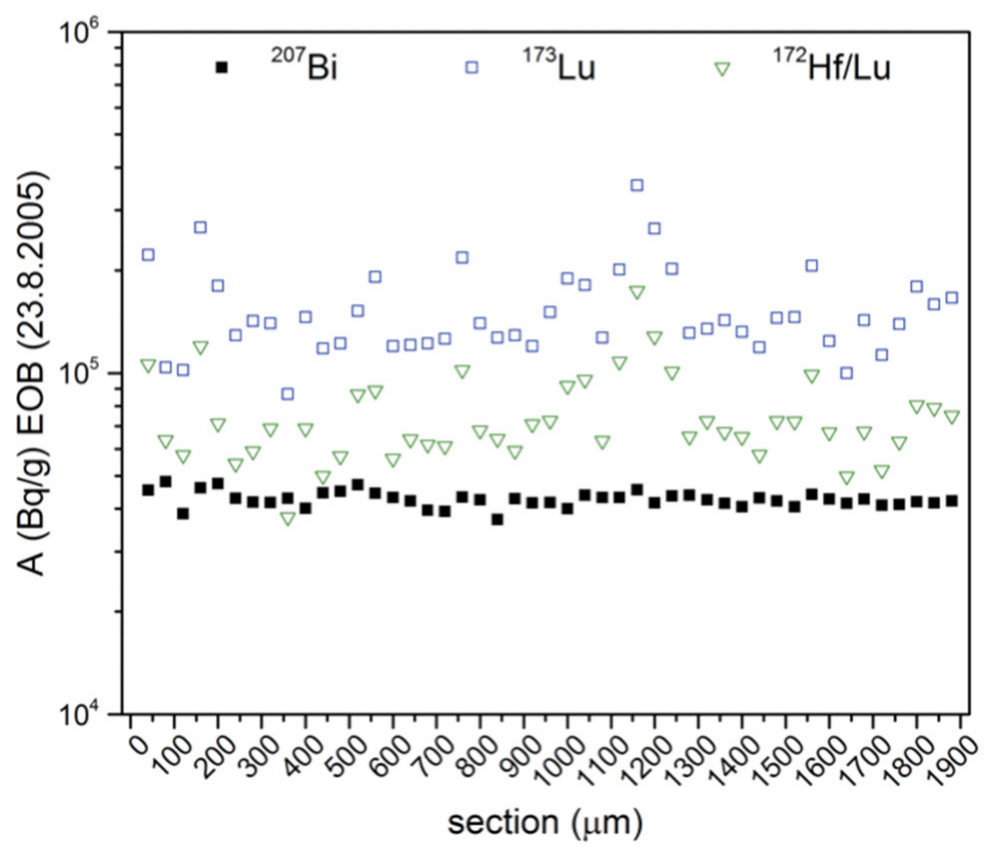

Fig. 10. Distribution of ${ }^{207} \mathrm{Bi},{ }^{173} \mathrm{Lu}$ and ${ }^{172} \mathrm{Hf} / \mathrm{Lu}$ in sections cut from a bulk LBE sample obtained from segment T2-1H of the ISOLDE target.

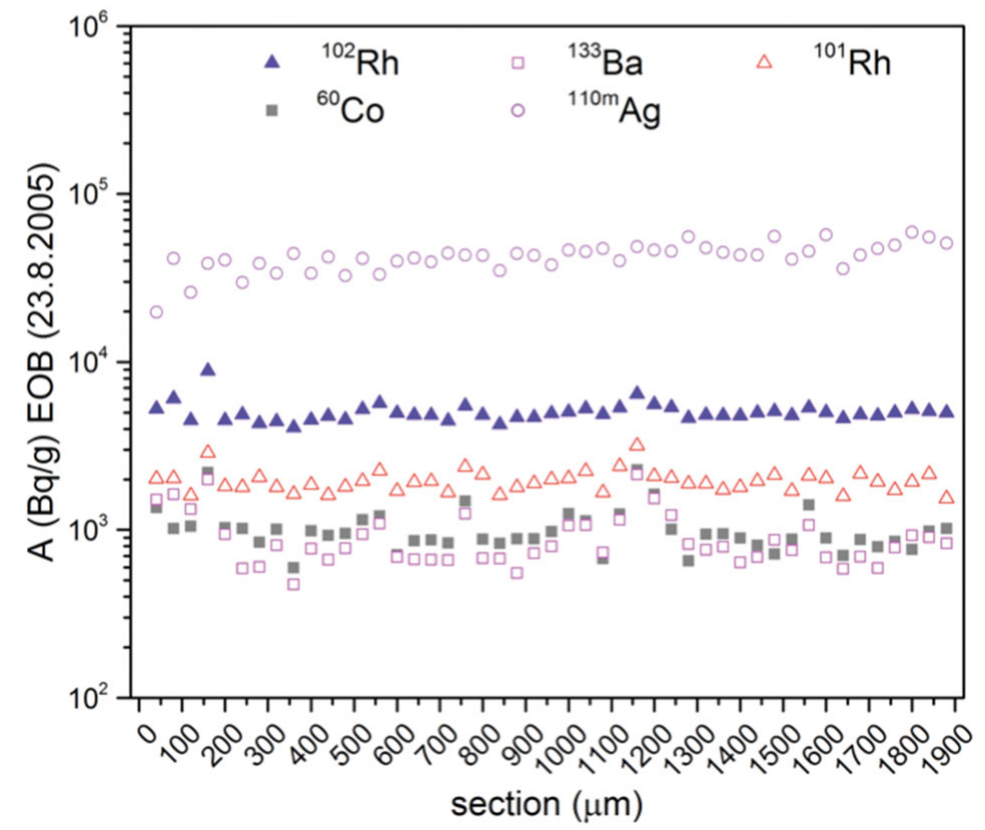

Fig. 11. Distribution of ${ }^{60} \mathrm{Co},{ }^{101} \mathrm{Rh},{ }^{102} \mathrm{Rh},{ }^{133} \mathrm{Ba}$, and ${ }^{110 m} \mathrm{Ag}$ in sections cut from a bulk LBE sample obtained from segment $\mathrm{T} 2-1 \mathrm{H}$ of the ISOLDE target.

All other nuclides show a significantly larger scatter in the distribution of their activity concentration throughout the set of sections, which is also reflected in the standard error of the mean value of the activity concentrations given in table 4 . It is noteworthy that there are several samples, such as those at distances of 160,760 and $1160 \mu \mathrm{m}$ from the starting point of the sectioning, which contain all radionuclides except from those of Bi and Ag at significantly higher concentrations compared to the remaining samples. This effect seems to be most pronounced for electropositive elements such as Lu, Hf and Ba, while it is less strong for the nobler Rh. Similarly, there are samples such as that taken at $360 \mu \mathrm{m}$ where the radionuclides of all studied chemical elements except for Bi and Ag are particularly low. Overall, there is a strong similarity in the distribution of $\mathrm{Lu}$, Hf, Ba, Co throughout the complete series of sections, indicating that the mechanism causing the inhomogeneity in the distribution is similar for these elements. 
Table 4. Averaged activity concentrations and their standard error for radionuclides detected in the 47 sections cut from a bulk LBE sample using a microtome, compared to INCL4.6-Abla07 and FLUKA predictions. Activity concentrations are decay-corrected to End of Beam (21.08.2005).

\begin{tabular}{|c|c|c|c|}
\hline \multirow{2}{*}{ Nuclide } & $\begin{array}{c}\text { Mean measured } \\
\text { activity concentration } \\
(\mathrm{kBq} / \mathrm{g})\end{array}$ & $\begin{array}{c}\text { Predicted activity concentration } \\
\text { INCL4.6-Abla07/CINDER'90 } \\
(\mathrm{kBq} / \mathrm{g})\end{array}$ & $\begin{array}{c}\text { Predicted activity concentration } \\
\text { FLUKA/ORIHET3 } \\
(\mathrm{kBq} / \mathrm{g})\end{array}$ \\
\hline${ }^{60} \mathrm{Co}$ & $1.02 \pm 0.33$ & 1.23 & 2.16 \\
\hline${ }^{101} \mathrm{Rh}$ & $1.96 \pm 0.31$ & 9.78 & 3.81 \\
\hline${ }^{102} \mathrm{Rh}$ & $5.0 \pm 0.7$ & 7.33 & 1.54 \\
\hline${ }^{110 m} \mathrm{Ag}$ & $43 \pm 7$ & & 113 \\
\hline${ }^{133} \mathrm{Ba}$ & $0.91 \pm 0.35$ & 0.43 & 122 \\
\hline${ }^{172} \mathrm{Hf} / \mathrm{Lu}$ & $75 \pm 23$ & 146.23 & 36.2 \\
\hline${ }^{173} \mathrm{Lu}$ & $155 \pm 49$ & 124.43 & \\
\hline${ }^{207} \mathrm{Bi}$ & $42.6 \pm 2.2$ & 36.24 & \\
\hline
\end{tabular}

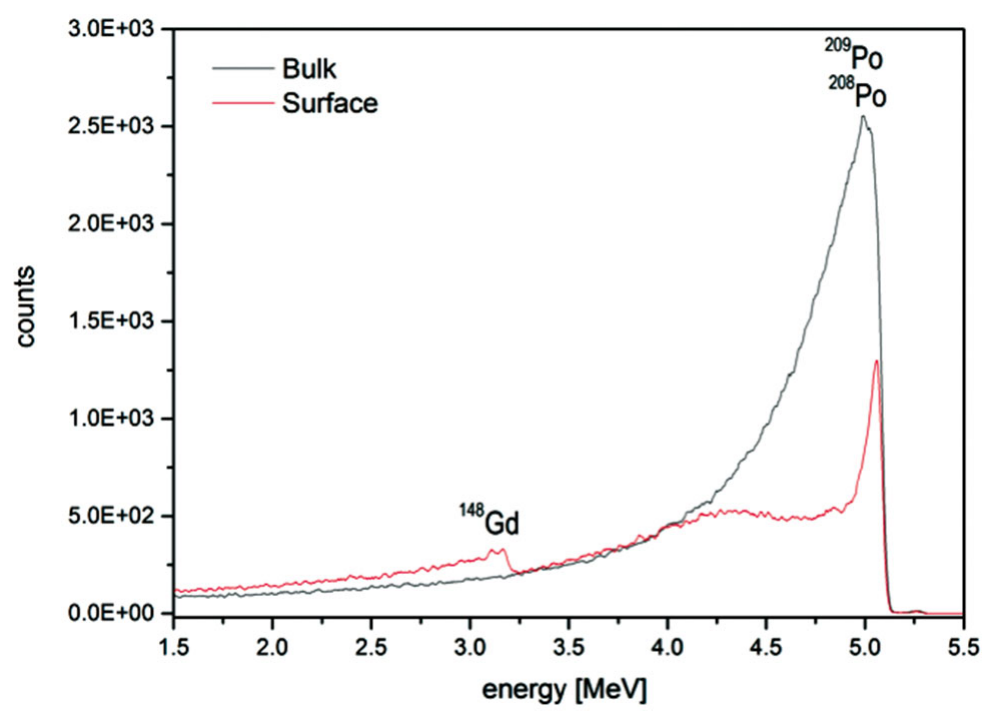

Fig. 12. Comparison of $\alpha$-spectra recorded from the bulk and surface side of sample T2-1C.

\subsection{Investigation of the surface enrichment in samples from the ISOLDE target}

Figure 12 shows a comparison of the $\alpha$-spectra recorded from two sides of sample $\mathrm{T} 2-1 \mathrm{C}$, one side representing the free surface of the LBE that formed the interface of LBE and vacuum during irradiation and the other side representing a fresh cut in the bulk LBE produced during sample taking. The spectrum recorded from the free surface clearly shows a signal of the $\alpha$-particles of approximately $3.2 \mathrm{MeV}$ emitted by ${ }^{148} \mathrm{Gd}$, while the spectrum taken from the bulk side of the sample does not show a significant peak at this energy. This indicates that ${ }^{148} \mathrm{Gd}$ was accumulated on the free surface during irradiation of the target, when the LBE was liquid. We want to point out that polonium, which is shown in figs. 12 and 14, will be the topic of another paper and is not discussed here.

The process of accumulation of radionuclides on the surface of liquid LBE was further studied by comparing the $\gamma$-spectra taken from a bulk LBE sample (T1-1C) from the ISOLDE target before and after applying the melting procedure described in the experimental sect. 2.4. After the melting, the surface scraped from the sample and the remaining LBE were analysed separately. Table 5 compiles the results of this investigation. The data obtained from the complete LBE sample before and after melting (columns 3 and 4, respectively) are in good agreement with the data obtained from other samples (tables 3 and 4). The changes caused by the melting are not very obvious when comparing columns 3 and 4 because the $\gamma$-rays emitted by the studied nuclides are rather penetrative and thus the sensitivity to surface effects is rather low when comparing data of the complete samples before and after melting. However, when we compare the data measured before the melting with those obtained from the $\gamma$-spectra recorded from the material scraped from the surface of the sample after melting (column 5) and those obtained from the remaining LBE 
Table 5. Activity concentrations of selected nuclides in samples T1-1C-bulk, determined by $\gamma$-spectrometry. Values are decaycorrected to End of Beam (23.08.2005). (T1-C-bulk = crude LBE, $a 1=$ after melting, $R=$ bulk, $A=$ surface).

\begin{tabular}{|c|c|c|c|c|c|}
\hline & $\begin{array}{c}\gamma \text {-energy } \\
(\mathrm{keV})\end{array}$ & $\begin{array}{c}\text { T1-1C-bulk } \\
m=238.7 \mathrm{mg} \\
A_{\mathrm{conc}} \pm 1 \sigma \\
(\mathrm{Bq} / \mathrm{g})\end{array}$ & $\begin{array}{c}\text { T1-1C-bulk_a1 } \\
m=238.23 \mathrm{mg} \\
A_{\text {conc }} \pm 1 \sigma \\
(\mathrm{Bq} / \mathrm{g})\end{array}$ & $\begin{array}{c}\text { T1-1C-bulk_a1_A } \\
m=27.15 \mathrm{mg} \\
A_{\text {conc }} \pm 1 \sigma \\
(\mathrm{Bq} / \mathrm{g})\end{array}$ & $\begin{array}{c}\text { T1-1C-bulk_a1_R } \\
m=211.08 \mathrm{mg} \\
A_{\text {conc }} \pm 1 \sigma \\
(\mathrm{Bq} / \mathrm{g})\end{array}$ \\
\hline${ }^{60} \mathrm{Co}$ & 1332.5 & $(2.5 \pm 0.05) \times 10^{3}$ & $(2.0 \pm 0.06) \times 10^{3}$ & $(1.2 \pm 0.04) \times 10^{4}$ & $(8.0 \pm 0.5) \times 10^{2}$ \\
\hline${ }^{101} \mathrm{Rh}$ & 198 & $(1.2 \pm 0.03) \times 10^{3}$ & $(10 \pm 0.3) \times 10^{2}$ & $(3.1 \pm 0.2) \times 10^{3}$ & $(1.0 \pm 0.03) \times 10^{3}$ \\
\hline${ }^{102} \mathrm{Rh}$ & 475.06 & $(1.7 \pm 0.01) \times 10^{4}$ & $(1.4 \pm 0.01) \times 10^{4}$ & $(2.1 \pm 0.06) \times 10^{4}$ & $(1.3 \pm 0.01) \times 10^{4}$ \\
\hline${ }^{110 m} \mathrm{Ag}$ & 884 & $(7.0 \pm 1.05) \times 10^{4}$ & $(7.4 \pm 1.1) \times 10^{4}$ & $(1.0 \pm 0.3) \times 10^{5}$ & $(7.4 \pm 0.9) \times 10^{4}$ \\
\hline${ }^{133} \mathrm{Ba}$ & 356.02 & $(2.1 \pm 0.05) \times 10^{3}$ & $(1.7 \pm 0.09) \times 10^{3}$ & $(1.3 \pm 0.04) \times 10^{4}$ & $(5.90 .8) \times 10^{2}$ \\
\hline${ }^{172} \mathrm{Hf} / \mathrm{Lu}$ & 1093.61 & $(4.7 \pm 0.08) \times 10^{4}$ & $(7.3 \pm 1.2) \times 10^{4}$ & $(1.3 \pm 0.01) \times 10^{6}$ & $(2.9 \pm 0.05) \times 10^{4}$ \\
\hline${ }^{173} \mathrm{Lu}$ & 272.09 & $(1.0 \pm 0.02) \times 10^{5}$ & $(1.2 \pm 0.7) \times 10^{4}$ & $(2.1 \pm 0.02) \times 10^{6}$ & $(4.9 \pm 0.4) \times 10^{4}$ \\
\hline${ }^{207} \mathrm{Bi}$ & 569.7 & $(1.3 \pm 0.009) \times 10^{5}$ & $(1.0 \pm 0.007) \times 10^{5}$ & $(1.0 \pm 0.009) \times 10^{5}$ & $(1.1 \pm 0.008) \times 10^{5}$ \\
\hline
\end{tabular}

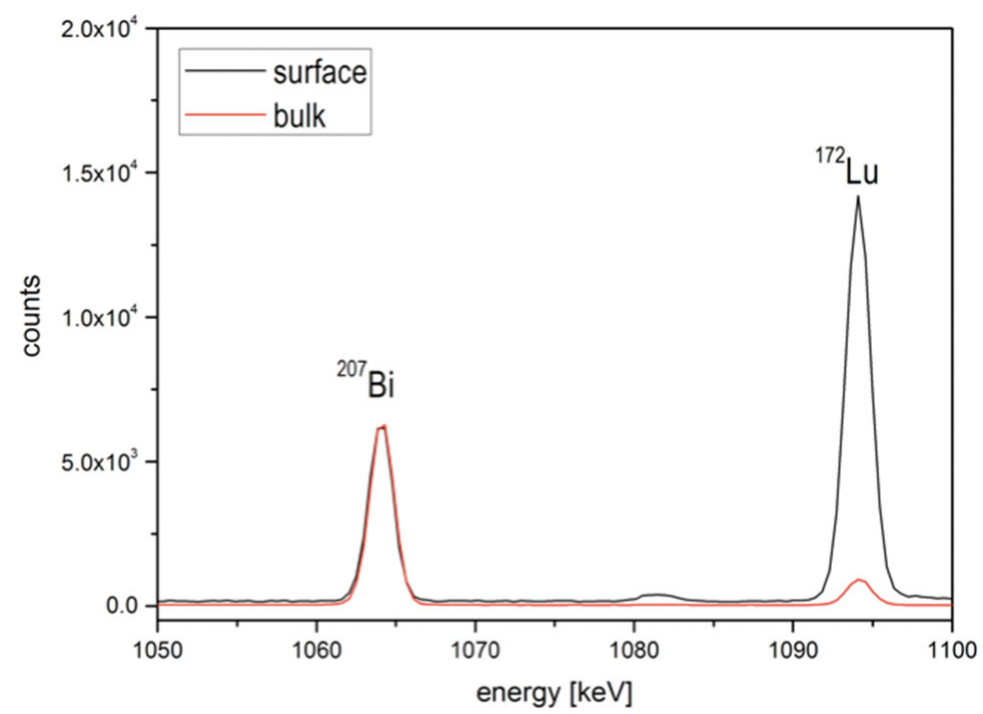

Fig. 13. Comparison of a section of the $\gamma$-spectra of the surface and bulk part of the LBE sample T1-1C-bulk from the ISOLDE LBE target after melting. The results of the $\gamma$-spectrometry indicate that lutetium tends to accumulate at the surface of the metal when molten.

(column 6), it becomes clear that a strong surface accumulation took place during the melting. The strongest effects are observed for the electropositive metals $\mathrm{Lu}, \mathrm{Hf}, \mathrm{Ba}$ and $\mathrm{Co}$, while the effect is less pronounced for the Rh isotopes. Consistent with the surface enrichment deduced from the data in column 5 , the nuclides that are found to accumulate on the surface are depleted in the remaining LBE (column 6). For ${ }^{110 m} \mathrm{Ag}$ no clear enrichment effect can be deduced within the limits of the experimental uncertainties. The same holds also for the matrix nuclide ${ }^{207} \mathrm{Bi}$. The effect of surface accumulation is also clearly visualized in a comparison plot of a section of the $\gamma$-spectra of the surface part removed after melting and the remaining bulk LBE (fig. 13).

Figure 14 shows a comparison of the $\alpha$-spectra recorded from sample T1-1C before and after melting. Since the $\alpha$-rays emitted by ${ }^{148} \mathrm{Gd}$ only have a range of a few $\mu \mathrm{m}$ in LBE, these measurements are very sensitive to surface effects. In the $\alpha$-spectrum recorded after the sample was molten, a sharp peak appears at $3.2 \mathrm{MeV}$, indicating that this nuclide accumulated at the surface during melting.

\subsection{Results on the ${ }^{148} \mathrm{Gd},{ }^{173} \mathrm{Lu}$ and ${ }^{146} \mathrm{Pm}$ content in MEGAPIE LBE/cover gas-interface samples}

All four samples analyzed from the LBE/cover gas-interface of the MEGAPIE target were not single phase but rather consisted of LBE contaminated with another solid material (in the following referred to as "deposit") in varying 


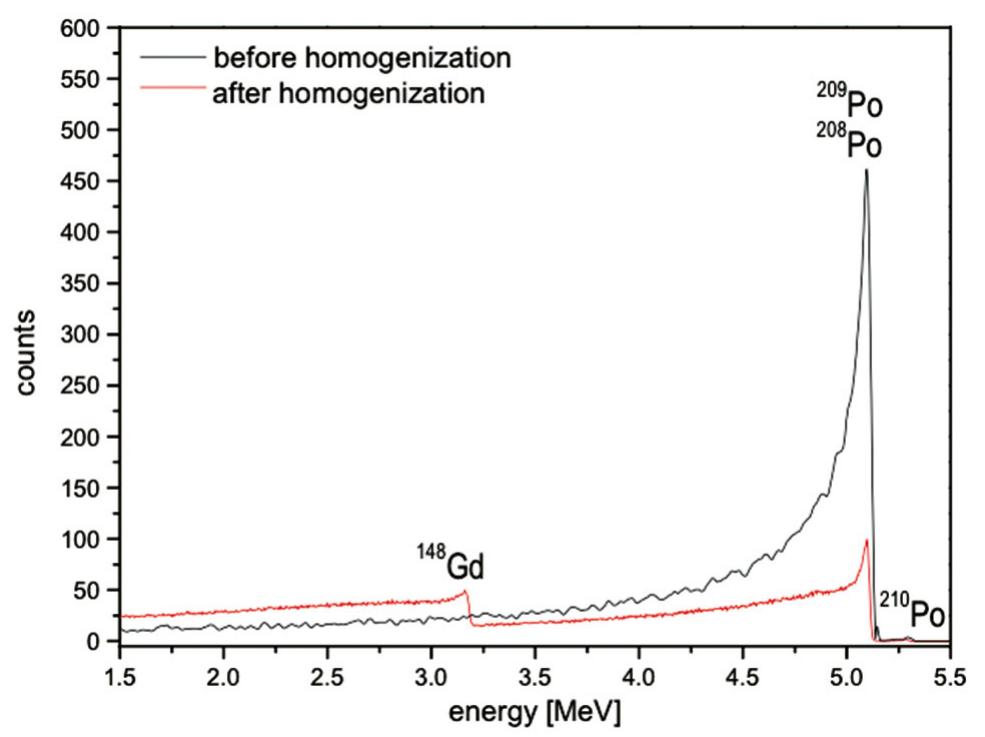

Fig. 14. Comparison of a section of the $\alpha$-spectra recorded from the LBE sample T1-1C before and after melting. The results indicate that gadolinium, similar to the chemically very similar lutetium, tends to accumulate at the surface of the molten metal.

proportions (figs. 5-8 and table 2). From $\gamma$-spectrometric analyses [3] it was concluded that the deposit contaminating the LBE is enriched in electropositive nuclear reaction products. In the following, we will study these accumulation effects in more detail for the lanthanide nuclides ${ }^{148} \mathrm{Gd},{ }^{173} \mathrm{Lu}$ and ${ }^{146} \mathrm{Pm}$. Two of these nuclides, namely ${ }^{148} \mathrm{Gd}$ and ${ }^{173} \mathrm{Lu}$, have mother nuclides in their isobaric decay chain that contribute significantly to their production, i.e. ${ }^{148} \mathrm{~Tb}$ and ${ }^{173} \mathrm{Hf}$ with half-lives of 23.6 and $1 \mathrm{~h}$, respectively. Since these half-lives are very short compared to the target operation time $(123 \mathrm{~d})$, the mother nuclides will practically completely decay to ${ }^{148} \mathrm{Gd}$ and ${ }^{173} \mathrm{Lu}$ during the irradiation. Furthermore, analyzing the deposition of ${ }^{172} \mathrm{Hf}$ it was found that Hf shows a deposition behavior that is undistinguishable from that of the lanthanide elements [7]. Finally, ${ }^{148} \mathrm{~Tb}$ is a lanthanide itself and will behave similar as its daughter ${ }^{148} \mathrm{Gd}$. Therefore, it is not possible to draw conclusions concerning the behavior of the mother nuclides ${ }^{148} \mathrm{~Tb}$ and ${ }^{173} \mathrm{Hf}$. Unfortunately, it was not possible to fully separate the deposit from the LBE by mechanical or chemical means. Thus, no explicit data on the radionuclide content of the pure LBE-free deposit could be obtained.

The results obtained for the four samples studied here are compiled in table 6 . Because of the multiphase nature of the samples we cannot provide activity concentrations. Therefore, the absolute activities $A_{\exp }$ found in fragments of a mass of $m_{\text {analyzed }}$ are given for each sample together with the total mass of the retrieved sample, $m_{\text {sample }}$. If we look at the ratios $A_{\text {exp }} / m_{\text {analyzed }}$ for ${ }^{173} \mathrm{Lu}$ in the free surface samples and compare it to the activity concentration found in the bulk samples $\left((2.7 \pm 0.7) \times 10^{4} \mathrm{~Bq}\right.$ per gram [5], it becomes clear that this isotope is strongly enriched in all samples from the LBE/cover gas-interface. The strongest enrichment, of a factor of about 5000 compared to the bulk activity concentration, is found in the grey and yellow colored material sticking to the "central rod" of the target (H08-U1-b), while the lowest enrichment (factor of 40) is found in the core drill from the free surface (H07-S). These results support the assumption that enrichment indeed occurs in the deposit, since the samples that mainly consist of LBE contaminated with the deposit (H07-S, H07-U2 and H08-U2) contain significantly less ${ }^{173}$ Lu compared to H08-U1-b. The latter sample rather contains the deposit in a concentrated form with lower amounts of LBE, as judged from visual inspection and from its rather brittle nature.

The largest activities per mass unit are consistently found in the sample H08-U1-b for all the three nuclides ${ }^{148} \mathrm{Gd}$, ${ }^{146} \mathrm{Pm}$ and ${ }^{173} \mathrm{Lu}$, confirming the very similar chemical behavior of the lanthanides.

Due to the rather undefined geometry of the samples and the unknown composition and dimension of the surface layer, a quantitative analysis is very difficult. In the following, we will roughly estimate from our analytical results the total amount of ${ }^{148} \mathrm{Gd},{ }^{146} \mathrm{Pm}$ and ${ }^{173} \mathrm{Lu}$ associated with the depositions in the top part of the target, corresponding to the free surface of LBE and its remainders sticking to the walls of construction materials. For this purpose, we will first estimate the activity of each nuclide associated to the four different free surface-related types of material retrieved from the target, using knowledge from construction drawings, visual inspection of the respective target sections during sampling and in some cases conservative assumptions.

- For the sample taken by core drilling in the solidified LBE free surface itself (H07-S) we know the area of the top surface of the cylinder obtained by core drilling (fig. 8, table 2). By relating this area $\left(3.14 \times 10^{-6} \mathrm{~m}^{2}\right)$ to the surface area of the ring structure that contained the LBE free surface $\left(0.03 \mathrm{~m}^{2}\right)$ we can extrapolate to the activity contained in that ring structure by dividing the measured activity by the ratio of cylinder top surface to the total LBE free surface, $1.1 \times 10^{4}$. 


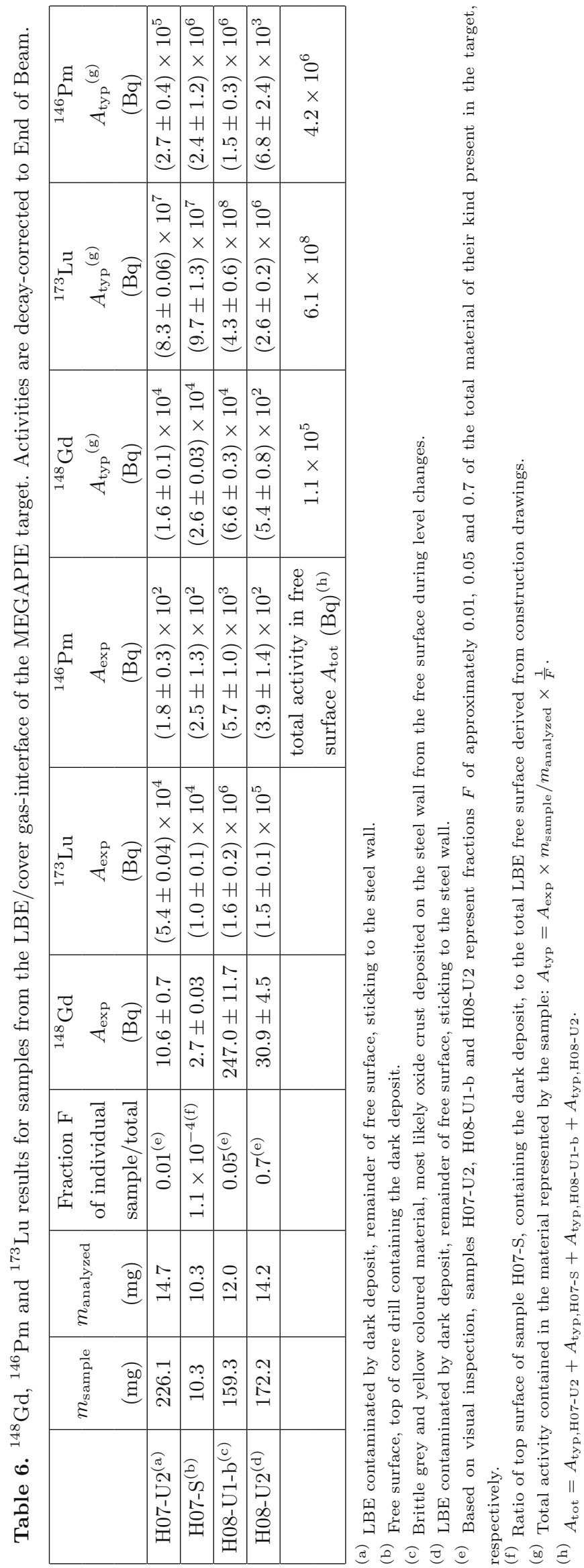


- For material similar to the sample H07-U2, we estimate from visual inspection during the sampling that we have retrieved about $10 \%$ of this LBE contaminated with the dark deposit from the ring structure it was located in. Sample H07-U2 itself constituted about $10 \%$ of the retrieved material, and thus to a fraction of 0.01 of the total material of this type present in the target. To estimate the amount of the studied nuclides in material of this type, we assumed that the analyzed part of the sample $(14.7 \mathrm{mg})$ is representative for the complete sample $(226.1 \mathrm{mg})$, and moreover also for the entire material of this kind present in the target. Then, the activity associated to this type of material can be estimated by dividing the activity found in the analyzed part of the sample by the mass ratio and the fraction estimated above.

- Sample H08-U1-b consisted of grey and yellow deposit sticking to the central rod located within the expansion volume of the target. From visual inspection we can state that we were able to retrieve the major part of this material from the target. For the further evaluation we will assume conservatively that the retrieved fraction was only $50 \%$, amounting to $1.6 \mathrm{~g}$. The sample studied here represents $10 \%$ of this material, and thus to a fraction of about 0.05 of the entire material of this type present in the target. The activity in this material was estimated, in a similar way as outlined above for sample H07-U2, assuming that the analyzed part of the sample $(12.0 \mathrm{mg})$ is representative for the complete sample $(159.3 \mathrm{mg})$ as well as for all the material of this type.

- There was only few material similar to sample H08-U2 found in the target, and it was completely retrieved in the sampling, amounting to $0.24 \mathrm{~g}$, sample H08-U2 representing roughly $70 \%$ of it, i.e. a fraction of 0.7 . The content of ${ }^{148} \mathrm{Gd},{ }^{146} \mathrm{Pm}$ and ${ }^{173} \mathrm{Lu}$ in the material of this type was estimated assuming that the analyzed part of the sample $(14.2 \mathrm{mg})$ is representative for all material of this type, as described above for samples H07-U2 and H08-U1-b.

The results of scaling up the analytical data to the content of ${ }^{148} \mathrm{Gd},{ }^{146} \mathrm{Pm}$ and ${ }^{173} \mathrm{Lu}$ in the individual types of materials are compiled in columns 8 to 10 of table 6 . Finally, the total activity of each nuclide associated to the LBE free surface and its remainders sticking to the walls is calculated as the sum over the four different types. This sum amounts to $1.1 \times 10^{5}, 6.1 \times 10^{8}$ and $4.2 \times 10^{6} \mathrm{~Bq}$ for the three nuclides ${ }^{148} \mathrm{Gd},{ }^{146} \mathrm{Pm}$ and ${ }^{173} \mathrm{Lu}$, respectively. These values correspond to 0.1 to $0.2 \%$ of the activities predicted by nuclear physics codes [5,28]. Since only one measurement per sample type exists, the assumption that the analyzed part of the samples is really representative for the entire amount of material of the respective type could be questioned. However, the values estimated are so low that we can certainly state that the contribution of ${ }^{148} \mathrm{Gd},{ }^{146} \mathrm{Pm}$ and ${ }^{173} \mathrm{Lu}$ located in the LBE free surface to the total amount of these nuclides in the entire MEGAPIE target is negligible.

\section{Discussion}

The fairly good agreement of activity concentrations measured in the bulk LBE of the ISOLDE target with predictions obtained from nuclear physics codes support the interpretation that the major fraction of all radionuclides studied here remained in the bulk during the irradiation of this target. However, the results of counting the $\alpha$-particles emitted from the free surface of the LBE indicates an enrichment of ${ }^{148} \mathrm{Gd}$ in this surface. A similar enrichment has also been observed in earlier studies comparing the ${ }^{172} \mathrm{Hf} / \mathrm{Lu}$ activity of samples taken from the bulk and the surface of the same ISOLDE target $[24,29]$. Apparently, the amount of the radionuclides accumulated in the free surface is not sufficient to achieve a significant depletion in the bulk LBE.

For the MEGAPIE target, the results presented here also prove an enrichment of lanthanide radionuclides in the free surface formed at the interface of LBE with the cover gas during irradiation. Other recent studies on samples from the MEGAPIE target [3,7] revealed a similar enrichment also for other radionuclides such as ${ }^{133} \mathrm{Ba},{ }^{173} \mathrm{Lu},{ }^{60} \mathrm{Co}$ and ${ }^{101,102} \mathrm{Rh}$. However, in contrast to the ISOLDE target, in the MEGAPIE target a strong depletion of lanthanides in the bulk LBE was detected [5]. Only about $10 \%$ of ${ }^{148} \mathrm{Gd},{ }^{146} \mathrm{Pm}$ and ${ }^{173} \mathrm{Lu}$ were found to remain in the bulk LBE, while the major part of these nuclides accumulate on the interface of the LBE with the construction materials, i.e. the walls of the target container. The results of the quantitative analysis of ${ }^{148} \mathrm{Gd},{ }^{146} \mathrm{Pm}$ and ${ }^{173} \mathrm{Lu}$ in the free surface samples of MEGAPIE reported in the present paper, together with the estimation of the total content of the free surface, indicate that though a significant enrichment of lanthanide nuclides is observed at the free surface of LBE in the MEGAPIE target, the total amounts of the radionuclides accumulated in this region are much smaller than those detected on the walls of the target. However, the extent of enrichment at the target walls and in the free surface is of similar size, and the fact that the total amount of radionuclides located in the free surface is much smaller than that on the target walls simply reflects that the free surface is orders of magnitude smaller than the inner surface of the target.

The fact that in the MEGAPIE target a significant depletion of those radionuclides accumulating on the surfaces occurs, while in the ISOLDE target no evidence for severe depletion was found, can be qualitatively explained by the very different characteristics of the two targets: The ISOLDE target had a very simple geometry with a rather low surface-to-volume ratio, and the molten LBE was stagnant during the operation (apart from shock induced by the impinging proton beam), while the MEGAPIE target had a very complex inner structure with a high surface-to-volume ratio (e.g. surfaces of the heat exchanger), and furthermore the LBE was continuously pumped at high speed. Thus, in 
contrast to the ISOLDE target, in the MEGAPIE target there is more surface available to accumulate radionuclides of those chemical elements that tend to separate from the liquid metal, and furthermore the transport processes involved in the accumulation are strongly enhanced by the pumping, both circumstances favoring accumulation on surfaces and depletion in the bulk. The results of the melting experiment performed in the present study on an LBE sample from the ISOLDE target show that there is indeed a pronounced tendency of ${ }^{133} \mathrm{Ba},{ }^{148} \mathrm{Gd},{ }^{173} \mathrm{Lu},{ }^{172} \mathrm{Hf} / \mathrm{Lu},{ }^{60} \mathrm{Co}$, and possibly to a lesser extent also ${ }^{101,102} \mathrm{Rh}$, to separate from the liquid LBE and to accumulate on its surface. This demonstrates that there is a driving force for the separation and accumulation process in the material as it was retrieved after irradiation, indicating that the separation process was simply not complete during the ISOLDE irradiation.

Finally, the results of the analysis of bulk LBE from the ISOLDE target revealed that the distribution of ${ }^{207} \mathrm{Bi}$ representing the target material and also ${ }^{110 m} \mathrm{Ag}$ in the material is practically homogeneous, while for ${ }^{133} \mathrm{Ba},{ }^{173} \mathrm{Lu}$, ${ }^{172} \mathrm{Hf} / \mathrm{Lu},{ }^{60} \mathrm{Co}$, and to a lesser extent also for ${ }^{101,102} \mathrm{Rh}$, a larger scatter of the activity concentrations is observed throughout the various bulk LBE samples studied. It is noteworthy that the inhomogeneity appears to be more pronounced for strongly electropositive metals $\left({ }^{133} \mathrm{Ba},{ }^{173} \mathrm{Lu},{ }^{172} \mathrm{Hf} / \mathrm{Lu},{ }^{60} \mathrm{Co}\right)$ and less pronounced $\left({ }^{101,102} \mathrm{Rh}\right)$ or completely absent $\left({ }^{110 m} \mathrm{Ag}\right)$ for nobler metals. Thus, the inhomogeneity displays a similar trend to that observed in the tendency of the nuclides of different chemical elements to separate from the liquid metal and enrich on the surface, indicating that similar physicochemical factors govern both surface enrichment and the formation of inhomogeneities. The correlation of the strength of these effects with the electronegativity of the elements supports earlier assumptions [3, $5,24,29]$ that the enrichment is driven by the accumulation of the respective elements in an oxide layer that exists (or is formed during operation of the target) on the free surface of the liquid target material, or in case of inhomogeneous distribution within the bulk, a similar accumulation on particles of oxidic nature occurs. The results presented in this paper for two very different target systems are consistent. However, they provide qualitative information only, and more information on the nature of the underlying processes and the extent to which these enrichment effects occur under various conditions remains to be studied in detail. Such studies are certainly important for the safe and reliable operation of nuclear facilities employing LBE as coolant and/or spallation material because such solid depositions may change heat transfer and corrosion resistance at surfaces, accumulation of radionuclides will lead to larger decay heat effects and larger radiation doses at certain locations, and finally the formation of suspended hard particles may lead to enhanced erosion. Indeed, fluid dynamics modelling studies related to such phenomena in LBE-cooled Accelerator-Driven Systems (ADS) have been initiated recently [30,31]. However, for the moment they are focused on fuel dispersion within the LBE in case of severe accidents and its consequences, and they do not incorporate chemical effects at the current stage. The results presented in the paper at hand indicate that effects occurring in the coolant itself are an interesting topic for further studies, and that the fundamental chemical processes have to be understood to be incorporated into future modeling studies. For this purpose it is highly desirable to study not only the radionuclide content but also the chemical nature of the deposits formed in LBE based nuclear systems.

\section{Summary and conclusions}

The results obtained by radiochemical analysis of radionuclides produced by high-energy proton irradiation in LBE used as spallation material are in reasonable agreement with predictions obtained from nuclear physics code. The results of various recently performed investigations indicate that the produced radionuclides do not necessarily remain dissolved in the irradiated LBE but tend to accumulate on the surface of the liquid metal, and as a consequence they can be depleted in the bulk LBE. The extent to which this occurs depends on the chemical nature of the radionuclides and on the characteristics of the spallation target system. In the present study, the behavior of various nuclides of different chemical elements in two largely different target systems have been discussed, and differences in their tendencies to be depleted in the bulk target material and to accumulate on surfaces have been qualitatively explained based on their chemical properties and differences in the two target systems.

The observed inhomogeneity in the distribution of radionuclides, in particular ${ }^{133} \mathrm{Ba},{ }^{173} \mathrm{Lu},{ }^{172} \mathrm{Hf} / \mathrm{Lu},{ }^{60} \mathrm{Co}$ and, to a lesser extent, ${ }^{101,102} \mathrm{Rh}$, has several consequences. For the radiochemical analysis of material from similar sources, due to the non-homogeneous distribution the reliable determination of the radionuclide inventory is rather complicated. Sampling only the bulk is not sufficient for most of the radionuclides. To achieve a representative sampling, a large number of interface samples have to be analyzed as well, covering all possible locations where depositions can occur. Additionally, for a precise investigation of these interface samples it is desirable to develop sampling procedures allowing retrieving samples of defined geometry. However, such sampling on highly irradiate material is complicated because of radioprotection issues. Practical consequences arising from the tendency for radionuclide accumulation for the operation of LBE based nuclear systems have been briefly discussed. Finally, neglecting the possible local enrichment of radionuclides in a large-scale facility like a neutron spallation source or an accelerator-driven system (ADS) can lead to erroneous evaluation of dose rates and thus, a wrong risk assessment. Moreover, enhanced damage of the structure material, due to the radiation — especially if $\alpha$-emitters like ${ }^{148} \mathrm{Gd}$ are involved — has to be taken into account. 


\section{References}

1. G.S. Bauer, Nucl. Instrum. Methods Phys. Res. Sect. A 463, 505 (2001).

2. M. Wohlmuther, W. Wagner, J. Nucl. Mater. 431, 10 (2012).

3. B. Hammer, J. Neuhausen, V. Boutellier, H.P. Linder, N. Shcherbina, M. Wohlmuther, A. Türler, D. Schumann, J. Nucl. Mater. 450, 278 (2014).

4. B. Hammer, D. Schumann, J. Neuhausen, M. Wohlmuther, A. Türler, Nucl. Data Sheets 119, 280 (2014).

5. B. Hammer, J. Neuhausen, V. Boutellier, M. Wohlmuther, A. Türler, D. Schumann, Anal. Chem. 87, 5656 (2015).

6. B. Hammer-Rotzler, J. Neuhausen, V. Boutellier, M. Wohlmuther, D. Schumann, A. Türler, Radichim. Acta 103, 745 (2015).

7. B. Hammer-Rotzler, Analysis of the nuclide inventory in MEGAPIE, a proton irradiated lead-bismuth eutectic spallation target, PhD thesis, University of Bern (2015).

8. J. Neuhausen, U. Köster, B. Eichler, Radiochim. Acta 92, 917 (2004).

9. J. Neuhausen, B. Eichler, Radiochim. Acta 93, 155 (2005).

10. J. Neuhausen, Nucl. Instrum. Methods Phys. Res., Sect. A 562, 702 (2006).

11. J. Neuhausen, B. Eichler, Radiochim. Acta 94, 239 (2006).

12. L. Zanini et al., AIP Conf. Proc. 769, 1525 (2005).

13. Y. Tall, Mesures de taux de production d'éléments gazeux et volatiles lors de réactions induites par des protons de 1 et $1,4 \mathrm{GeV}$ sur des cibles épaisses de plomb et plomb-bismuth liquides, $\mathrm{PhD}$ thesis, Université de Nantes (2008).

14. E. Noah, V. Boutellier, R. Brütsch, R. Catherall, D. Gavillet, J. Krbanjevic, H.P. Linder, M. Martin, J. Neuhausen, D. Schumann, T. Stora, L. Zanini, J. Nucl. Mater. 431, 60 (2012).

15. L. Zanini, U. Köster, J.C. David, Y. Tall, M. Andersson, K. Berg, S. Cormon, M. Fallot, Y. Foucher, H. Frånberg, F. Gröschel, A. Guertin, T. Kirchner, S. Leray, E. Manfrin, E. Noah, H. Ravn, T. Stora, N. Thiollière, M. Wohlmuther, Nucl. Data Sheets 119, e1 (2014).

16. K. Samec, Nucl. Technol. 162, 358 (2008).

17. MEGAPIE leads the way to waste transmutation - CERN Courier (2007).

18. C. Fazio, F. Gröschel, W. Wagner, K. Thomsen, B.L. Smith, R. Stieglitz, L. Zanini, A. Guertin, A. Cadiou, J. Henry, P. Agostini, Y. Dai, H. Heyck, S. Dementjev, S. Panebianco, A. Almazouzi, J. Eikenberg, A. Letourneau, J.C. Toussaint, A. Janett, C. Perret, S. Joray, J. Patorski, W. Leung, P. Meloni, P. Turroni, A. Zucchini, G. Benamati, J. Konys, T. Auger, A. Gessi, D. Gorse, I. Serre, A. Terlain, J.-B. Vogt, A. Batta, A. Class, X. Cheng, F. Fellmoser, M. Daubner, S. Gnieser, G. Grötzbach, R. Milenkovic, C. Latgé, J.U. Knebel, Nucl. Eng. Des. 238, 1471 (2008).

19. E. Bubelis, P. Coddington, K. Mikityuk, Ann. Nucl. Energy 35, 1284 (2008).

20. H. Heyck, F. Gröschel, S. Dementievs, W. Wagner, ICANS-XVIII 18th Meet. Int. Collab. Adv. Neutron Sources Dongguan Guangdong P R China April 25-27 200\%.

21. W.B. Wilson et al., Status of CINDER'90 Codes and Data, in Proceedings of the 4th Workshop on Simulating Accelerator Radiation Environments, September 13-16, 1998, Knoxville, Tennessee, USA (1998) pp. 69-79 and Los Alamos National Laboratory Report LA-UR-98-361 (1998).

22. A. Boudard, J. Cugnon, J.-C. David, S. Leray, D. Mancusi, Phys. Rev. C 87, 014606 (2013).

23. A. Kelic, M.V. Ricciardi, K.-H. Schmidt, in Proceedings of the Joint ICTP-IAEA Advanced Workshop on Model Codes for Spallation Reactions, ICTP Trieste, Italy, 4-8 February 2008, edited by D. Filges, S. Leray, Y. Yariv, A. Mengoni, A. Stanculescu, G. Mank (IAEA INDC(NDS)-530, Vienna, 2008) p. 181.

24. J. Neuhausen, D. Schumann, R. Dressler, B. Eichler, S. Heinitz, B. Hammer, F. von Rohr, L. Zanini, V. Boutellier, M. Rüthi, J. Eikenberg, E. Noah, in Proc. DAE-BRNS Symp. Nucl. Radiochem. NUCAR-2011 Visakhapatnam (2011) p. 44.

25. T.T. Bohlen, F. Cerutti, M.P.W. Chin, A. Fassò, A. Ferrari, P.G. Ortega, A. Mairani, P.R. Sala, G. Smirnov, V. Vlachoudis, Nucl. Data Sheets 120, 211 (2014).

26. A. Ferrari, P.R. Sala, A. Fassò, J. Ranft, FLUKA: a multi-particle transport code CERN-2005-10 (2005) INFN/TC_05/11, SLAC-R-773.

27. F. Atchison, H. Schaal, ORIHET3-Version 1.12, A Guide for Users (2001).

28. L. Zanini, J.-C. David, A.Y. Konobeyev, S. Panebianco, N. Thiolliere, PSI Report Nr 08-04 (Paul Scherrer Institute Villigen, Switzerland, 2008).

29. J. Neuhausen, B. Hammer, D. Schumann, L. Zanini, V. Boutellier, M. Rüthi, J. Eikenberg, E. Noah, Ann. Rep. Lab. Radio-Env. Chem. Uni. Bern PSI (2010) p. 35.

30. R. Li, X.-N. Chen, A. Rineiski, V. Moreau, Ann. Nucl. Energy 79, 31 (2015).

31. X.-N. Chen, R. Li, A. Rineiski, W. Jäger, Energy Convers. Manag. 91, 93 (2015). 